\title{
Tuning the Helical Structures of Wells-Dawson POMs based Hybrid Compounds by Using the Isomeric Ligands
}

Pei-Pei Zhu ${ }^{\mathrm{a}}$, Long-Jiang Sun ${ }^{\mathrm{a}}$, Ning Sheng ${ }^{\mathrm{b}}$, Jing-Quan Sha ${ }^{\mathrm{a}, \mathrm{b} *}$,

Guo-Dong Liu, ${ }^{\mathrm{b}}$ Lian Yu, ${ }^{\mathrm{a}}$ Hong-Bin Qiu ${ }^{\mathrm{a}-}$, Shu-Xian $\mathrm{Li}^{\mathrm{a}}$

${ }^{a}$ School of Pharmacy, Jiamusi University, Jiamusi, 154007, China

${ }^{b}$ Department of Chemistry and Chemical Engineering, Jining University, Qufu, 273155, China

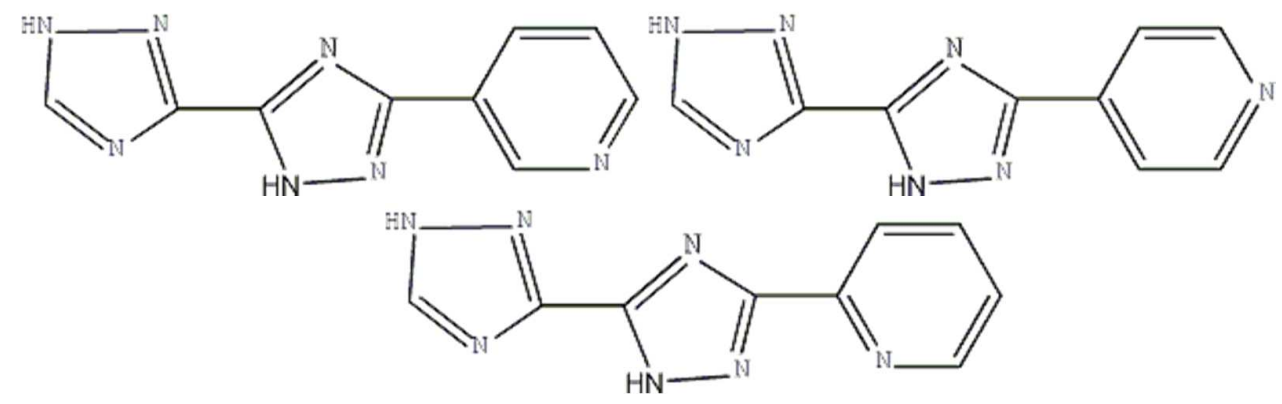

Scheme S1 Representations of the ligand $\mathrm{H}_{2}$ pyttz-I (3-(pyrid-3-yl)-5-(1H- 1,2,4-3-yl)1,2,4-triazolyl) , $\mathrm{H}_{2}$ pyttz-II 3-(pyrid-4-yl)-5-(1H- 1,2,4-3-yl)- 1,2,4-triazolyl and $\mathrm{H}_{2}$ pyttz-III (3-(pyrid-2-yl)-5-(1H- 1,2,4-3-yl)- 1,2,4-triazolyl.

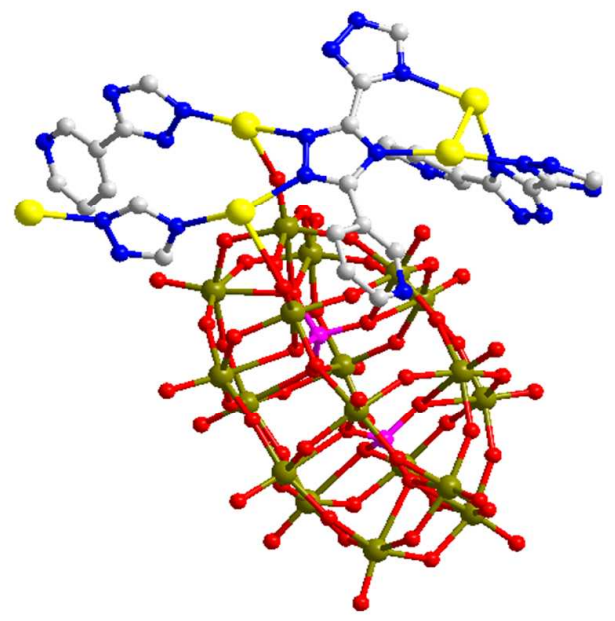

Fig.S1 Combined representation of the asymmetric unit of compound $\mathbf{1 .}$ 


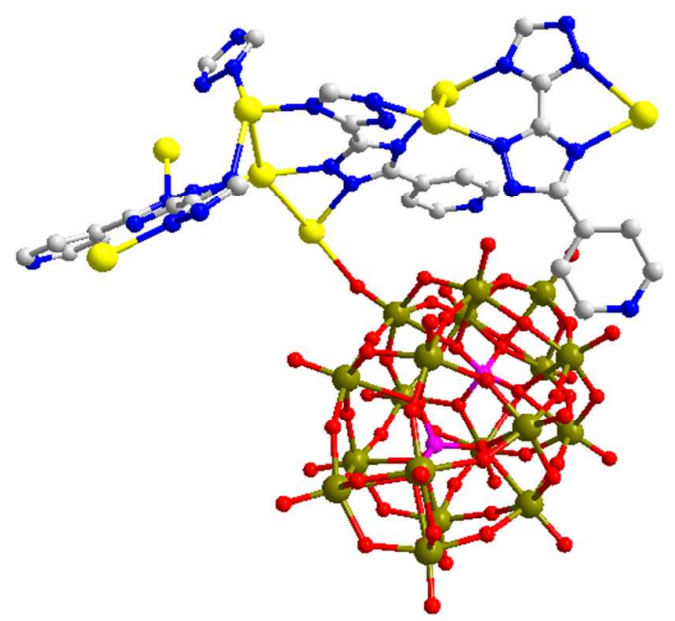

Fig.S2 Combined representation of the asymmetric unit of compound 2.

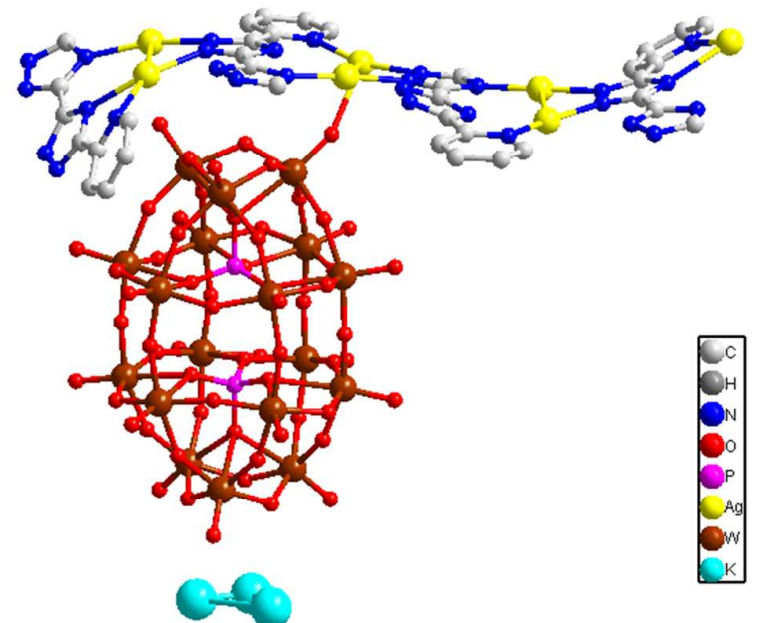

Fig.S3 Combined representation of the asymmetric unit of compound $\mathbf{3}$. 

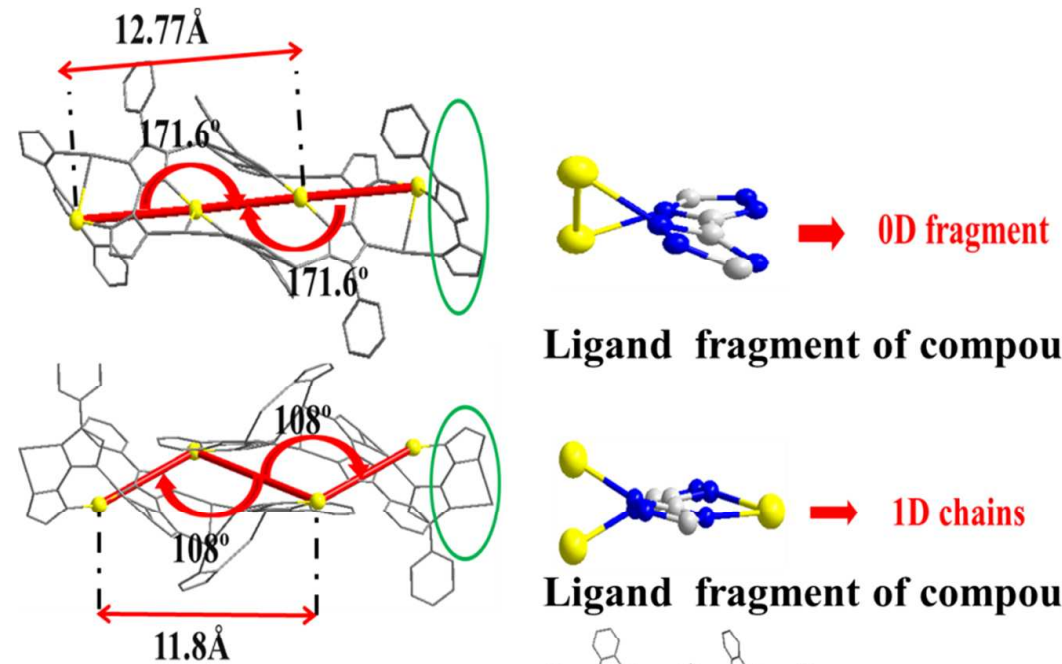

Ligand fragment of compound 1
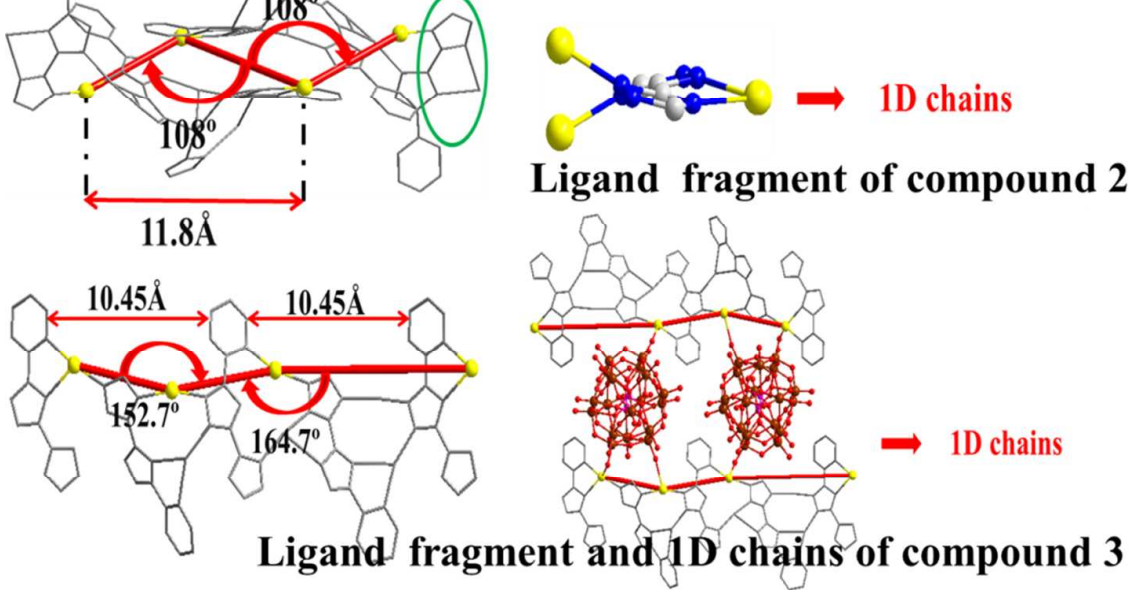

Fig.S4 The structure of Ag-pyttz subunits in compounds 1-3. 


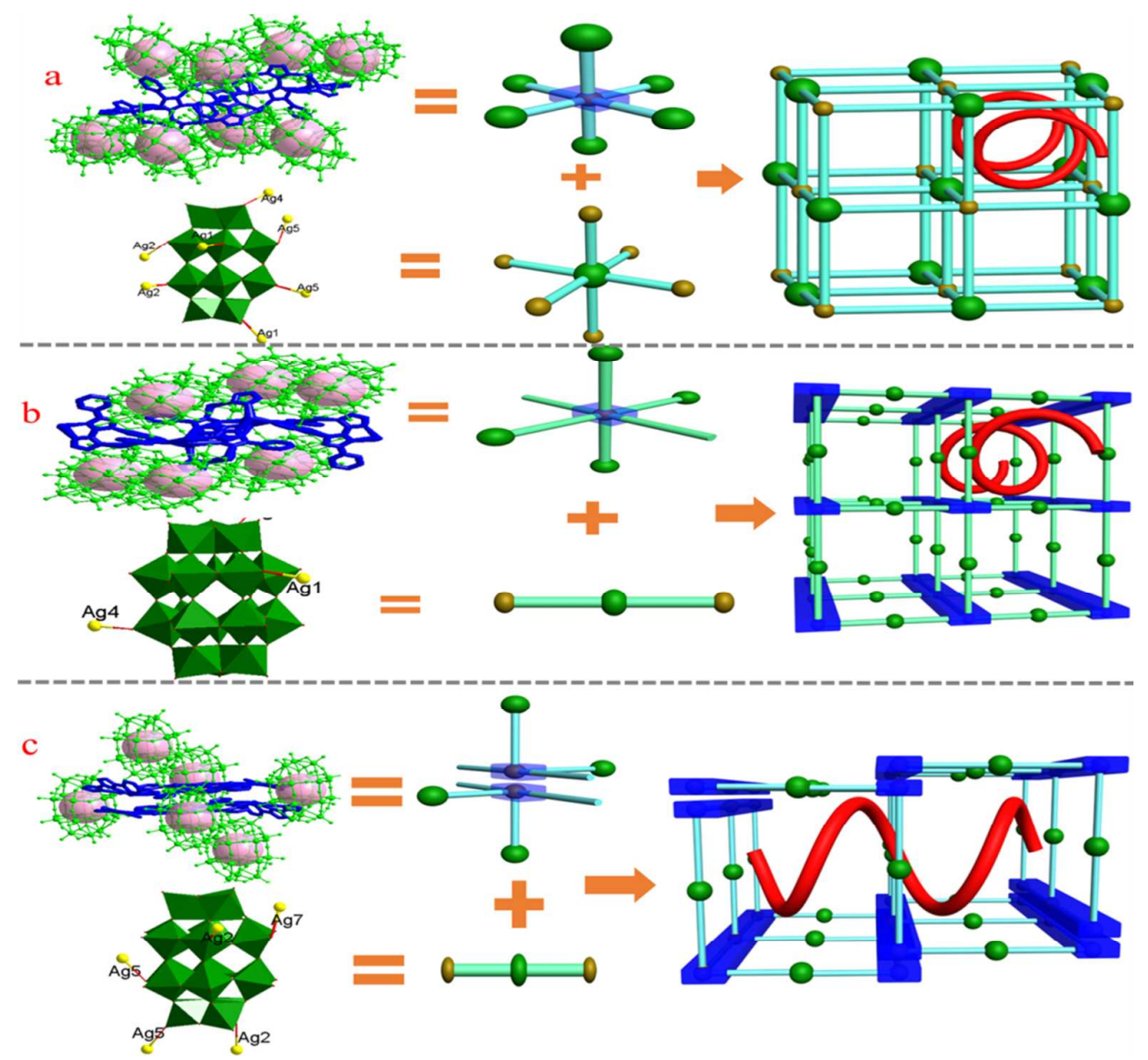

Fig.S5 Representations and formation of the whole structure and the role of each building block.
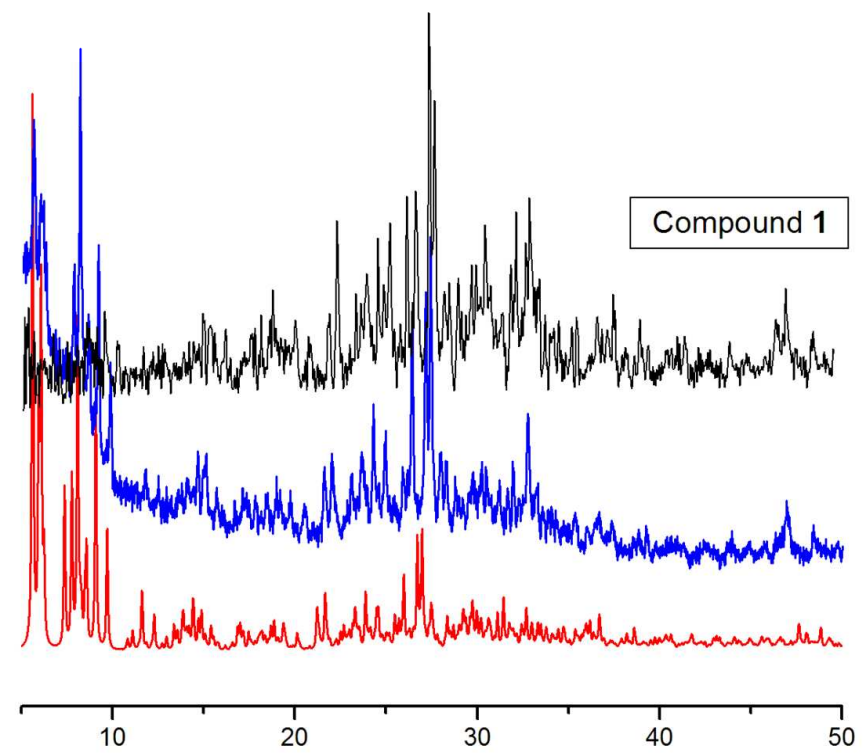

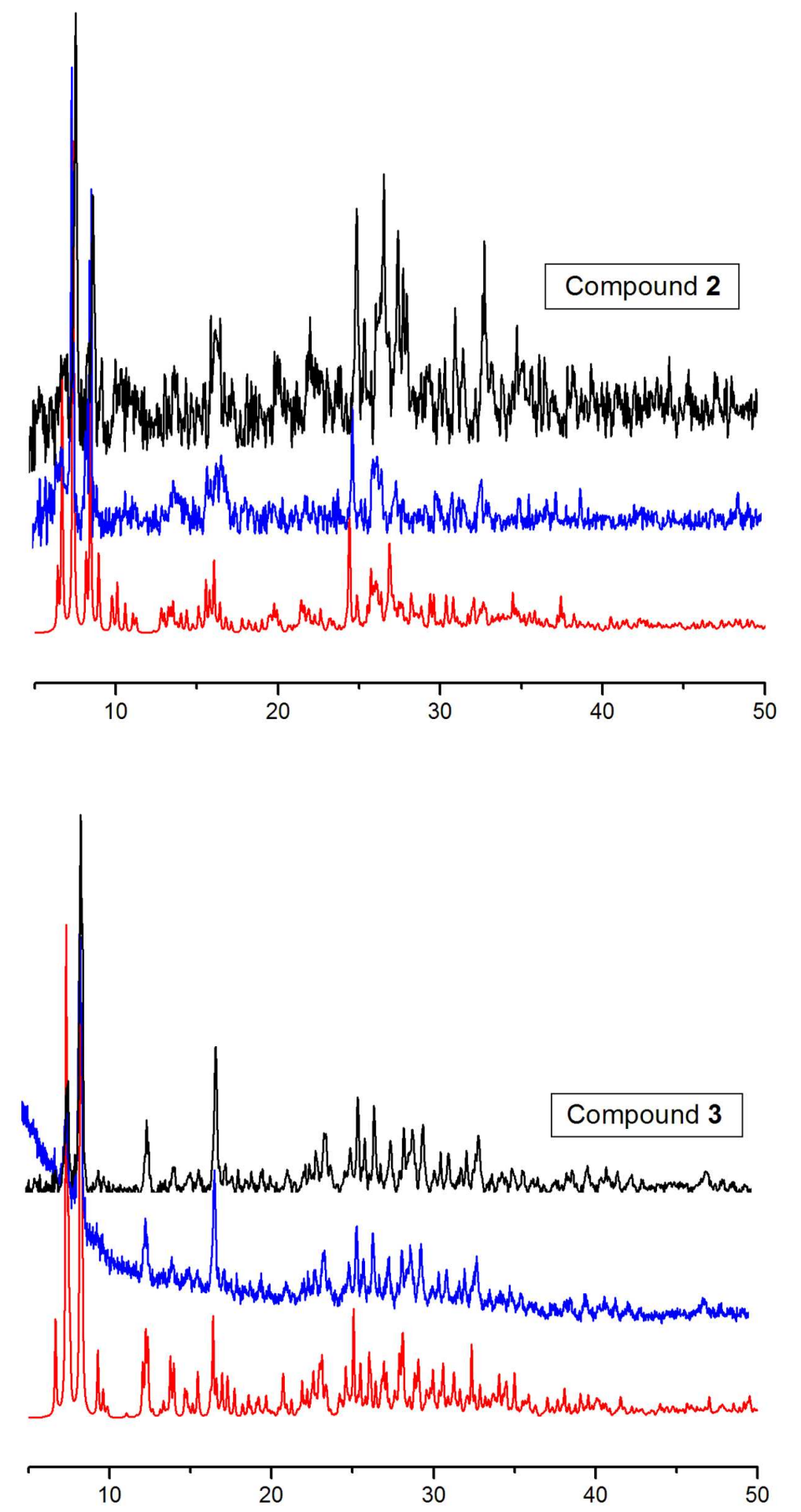

Fig.S6 XRD pattern for calculated, before and after photocatalytic reaction for Compounds 1-3 

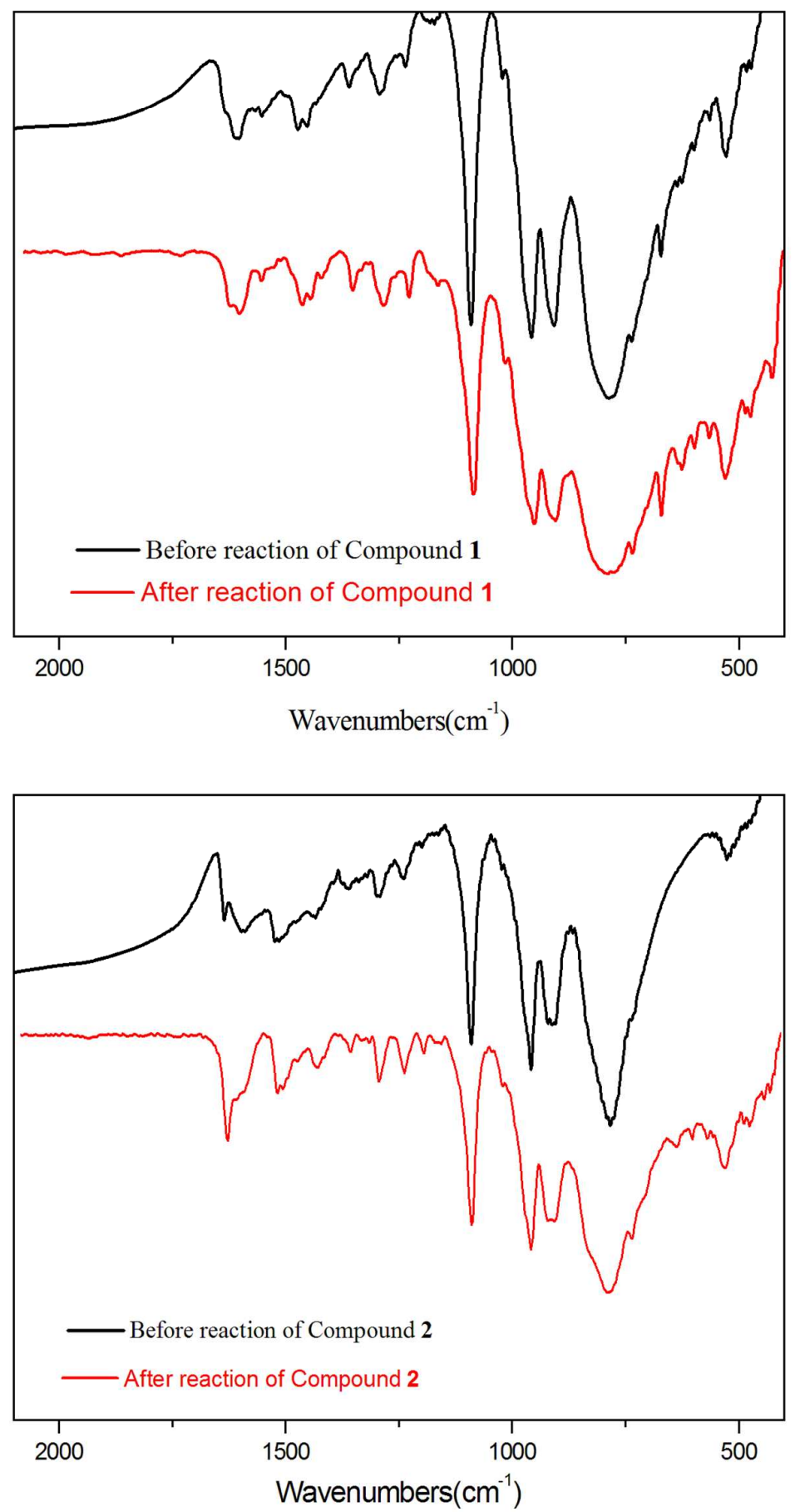


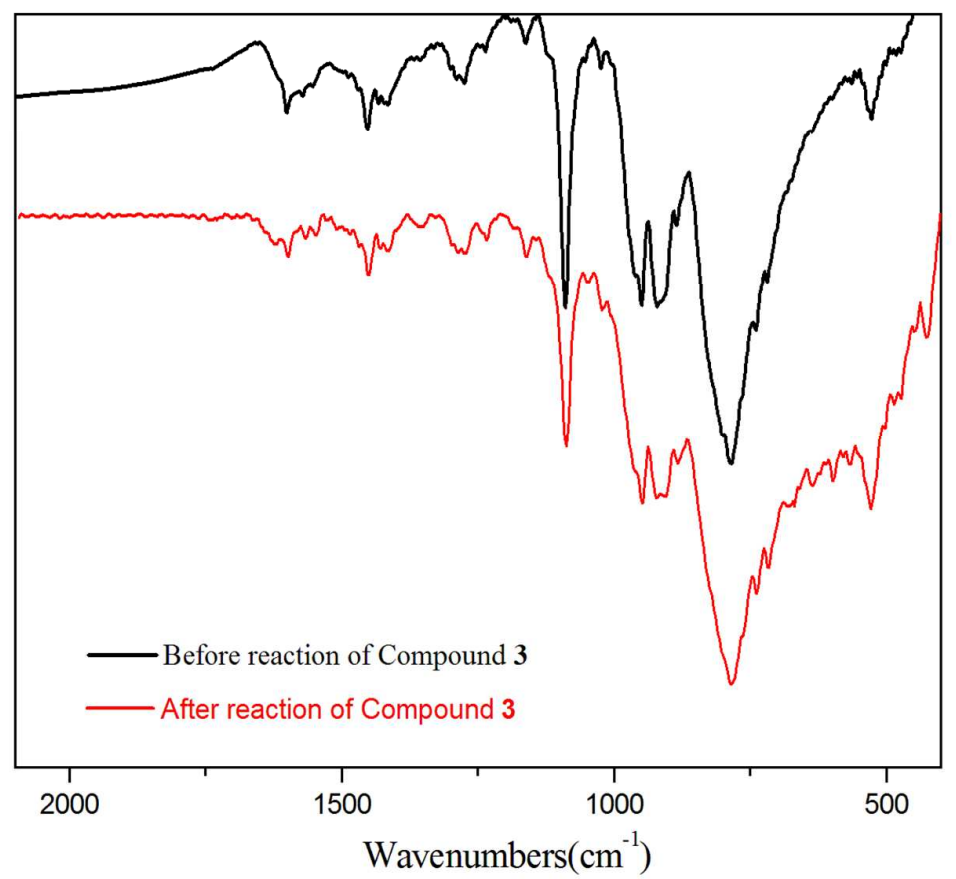

Fig.S7 IR spectra of compounds 1-3.

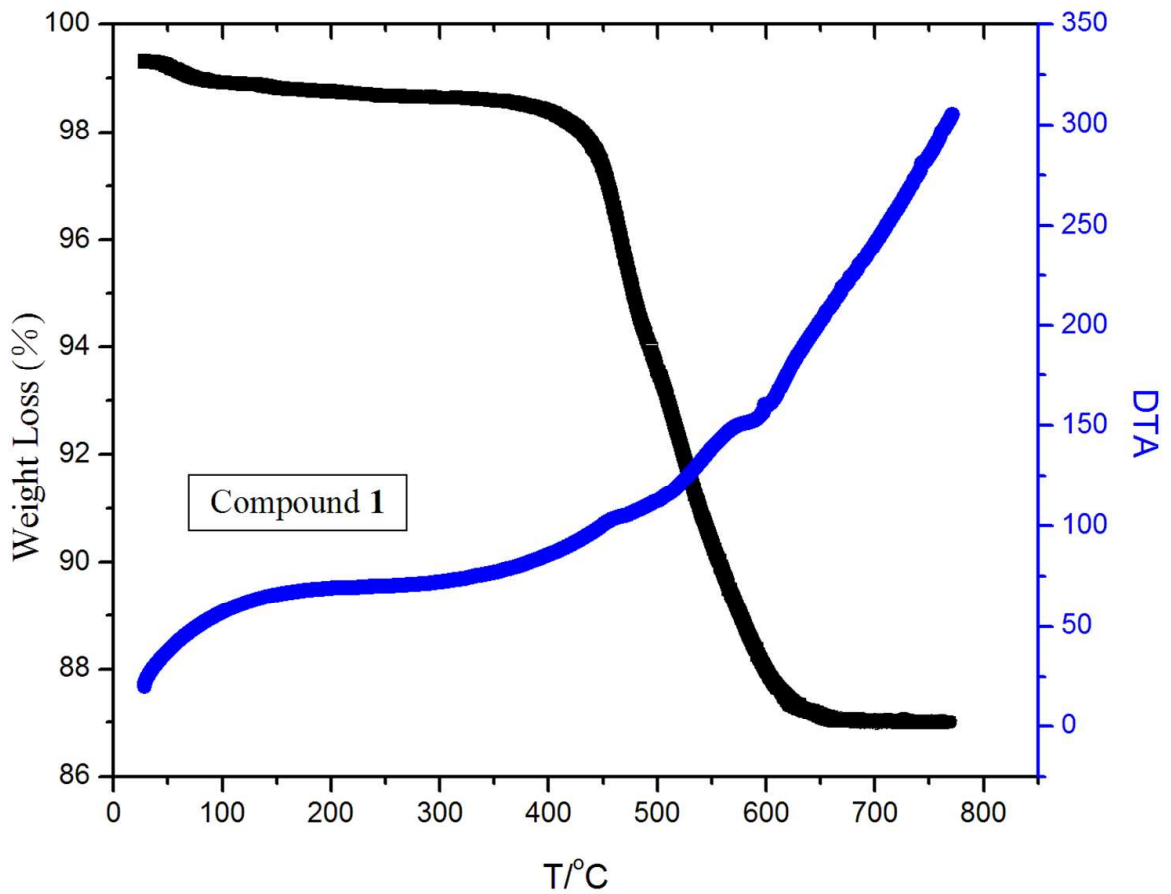



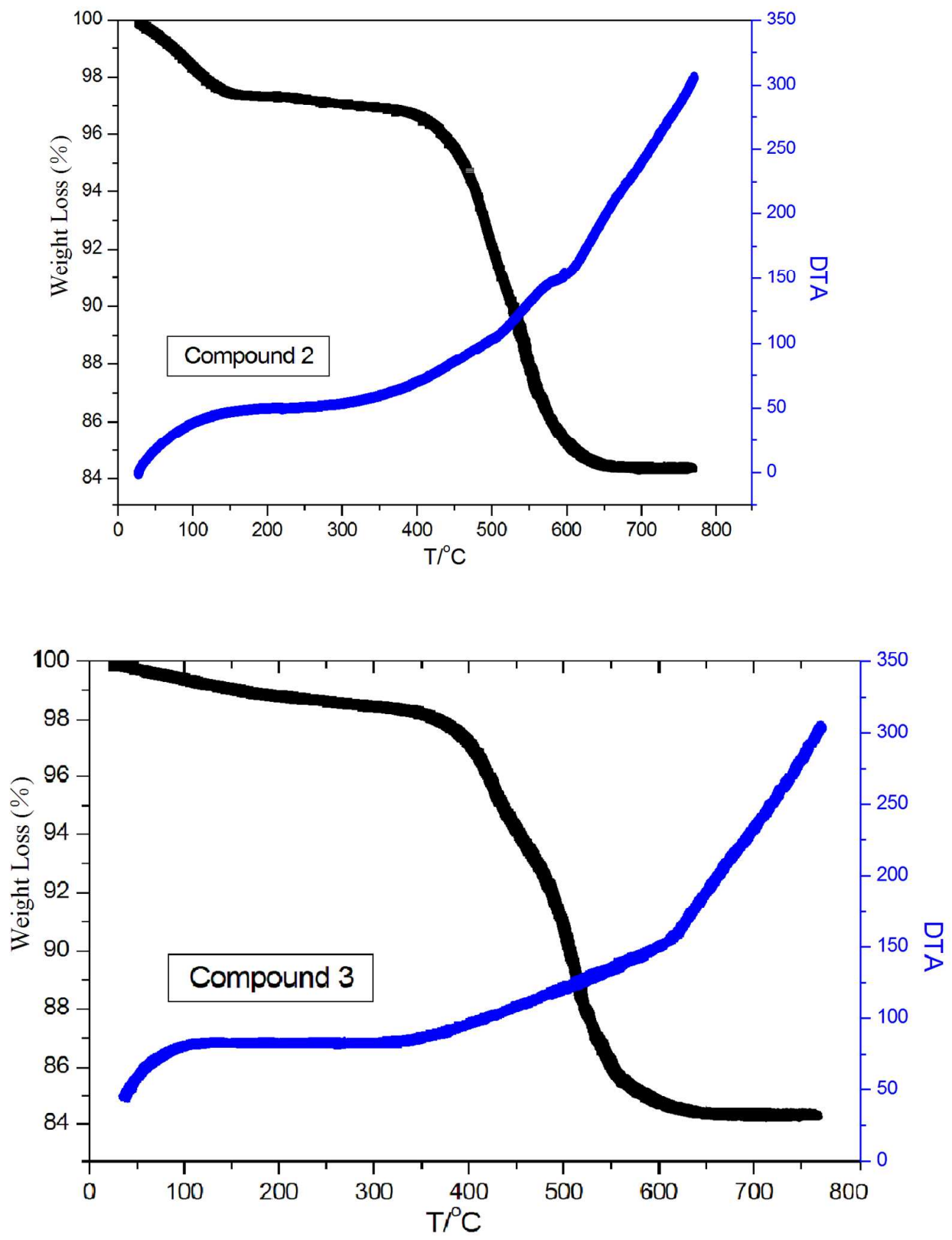

Fig.S8 TG-DTA curves of compounds 1-3. 

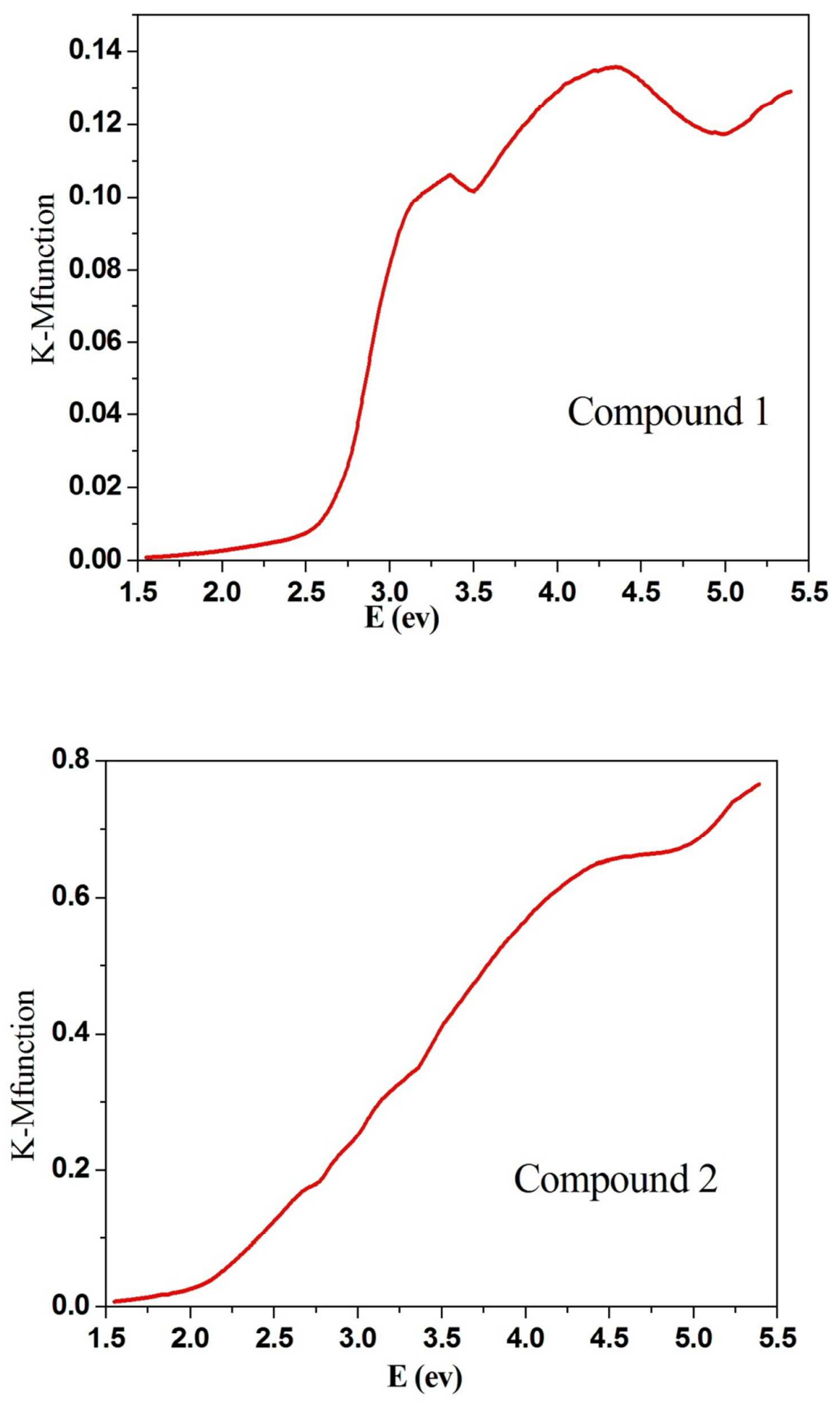


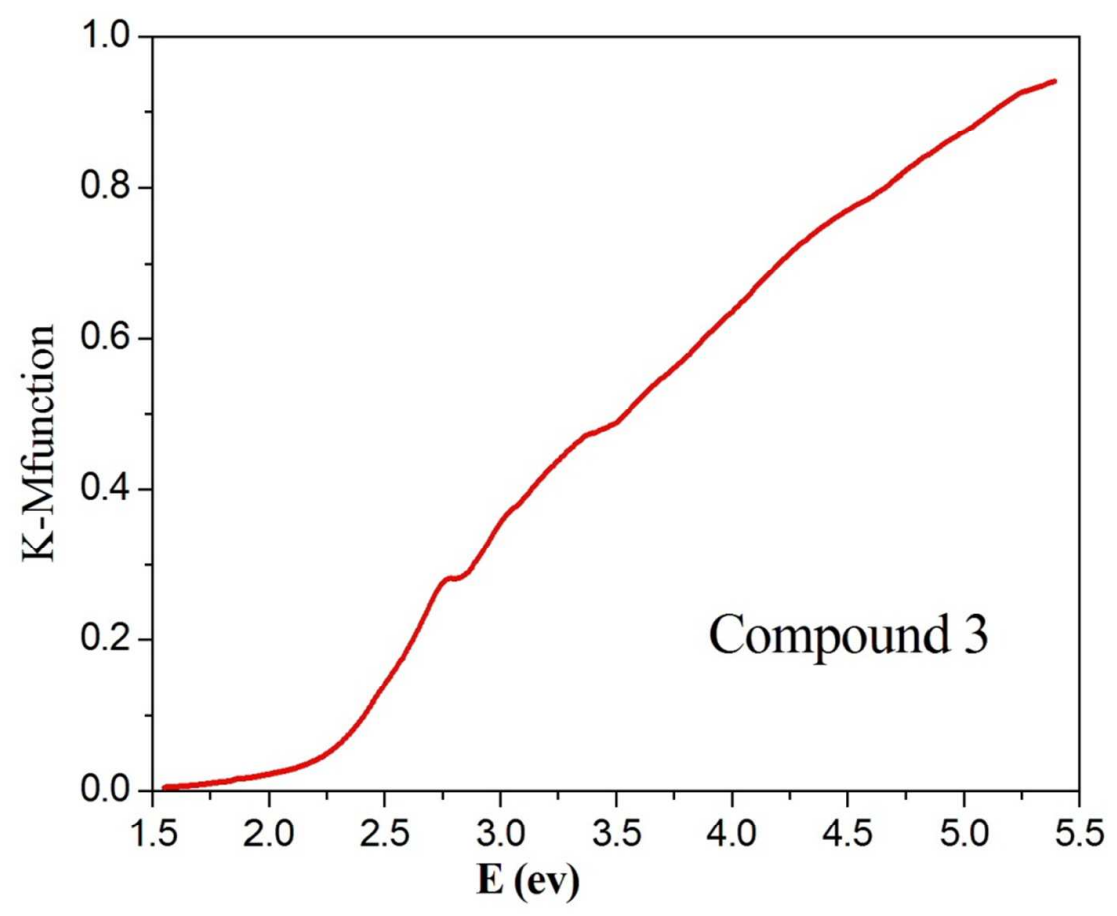

Fig.S9 The diffuse reflectance spectra of Compounds 1- 3 .

Table S1 Structural information of POMs, pyttz ligands and Ag ions

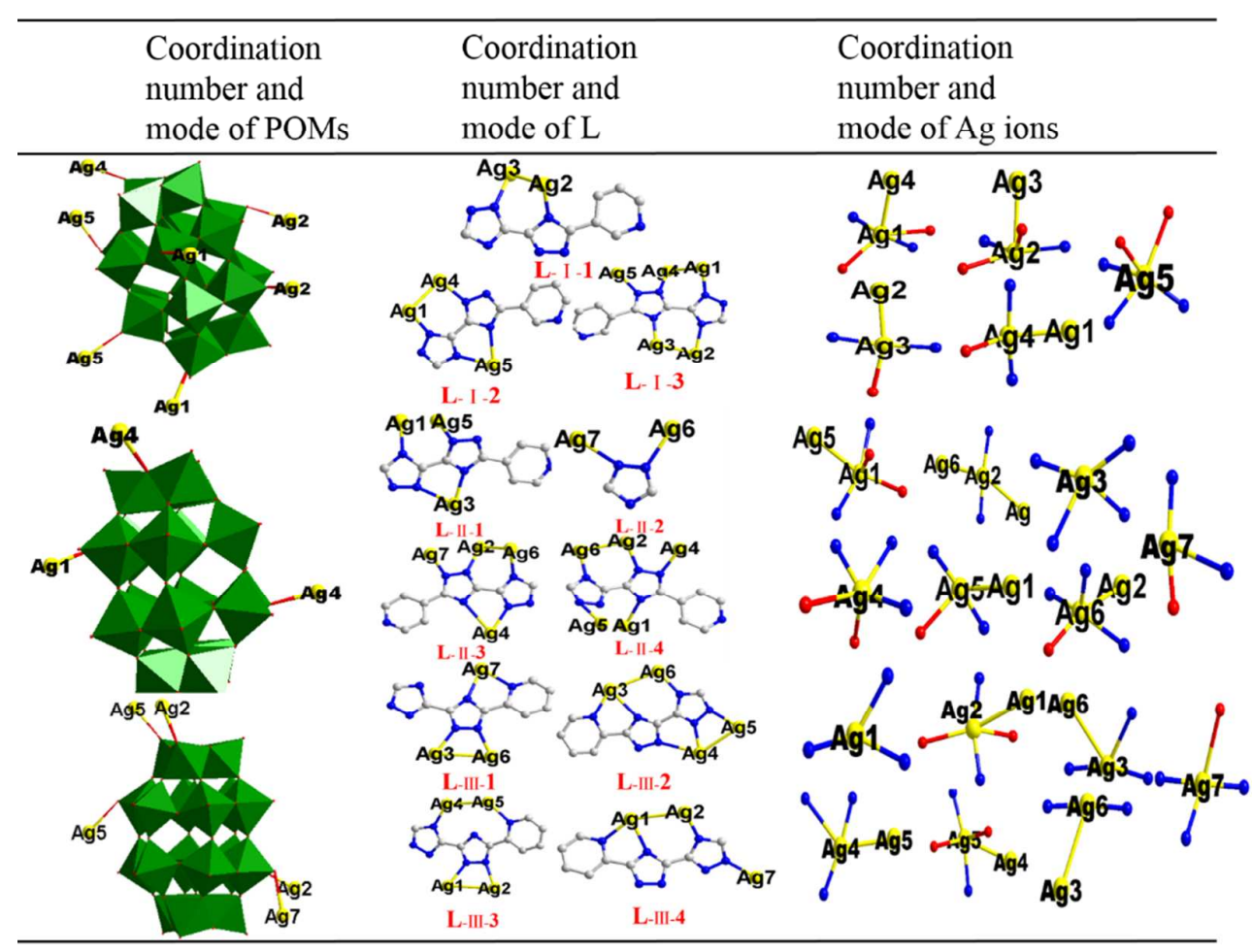


Table S2. Selected Bond Lengths $(\AA)$ and Bond Angles $\left({ }^{\circ}\right)$ for Compound 1.

\begin{tabular}{|c|c|c|c|}
\hline $\mathrm{N}(2)-\operatorname{Ag}(1)$ & $2.17(2)$ & $\mathrm{O}(33)-\mathrm{W}(1)$ & $1.706(14)$ \\
\hline $\mathrm{N}(4)-\operatorname{Ag}(5)$ & $2.213(19)$ & $\mathrm{O}(34)-\mathrm{W}(16)$ & $1.697(14)$ \\
\hline $\mathrm{N}(5)-\operatorname{Ag}(3)$ & $2.178(18)$ & $\mathrm{O}(34)-\operatorname{Ag}(4)$ & $2.585(15)$ \\
\hline $\mathrm{N}(6)-\operatorname{Ag}(4)$ & $2.18(2)$ & $\mathrm{O}(35)-\mathrm{W}(2)$ & $1.706(16)$ \\
\hline $\mathrm{N}(7)-\operatorname{Ag}(2)$ & $2.32(2)$ & $\mathrm{O}(36)-\mathrm{W}(17)$ & $1.707(13)$ \\
\hline $\mathrm{N}(8)-\operatorname{Ag}(4)$ & $2.095(19)$ & $\mathrm{O}(37)-\mathrm{W}(3)$ & $1.728(13)$ \\
\hline $\mathrm{N}(10)-\operatorname{Ag}(5)$ & $2.336(19)$ & $\mathrm{O}(38)-\mathrm{W}(15)$ & $1.911(14)$ \\
\hline $\mathrm{N}(11)-\operatorname{Ag}(1) \# 1$ & $2.16(2)$ & $\mathrm{O}(38)-\mathrm{W}(17)$ & $1.924(18)$ \\
\hline $\mathrm{N}(12)-\mathrm{Ag}(3)$ & $2.21(3)$ & $\mathrm{O}(39)-\mathrm{W}(7)$ & $1.891(14)$ \\
\hline $\mathrm{N}(13)-\operatorname{Ag}(2)$ & $2.22(2)$ & $\mathrm{O}(39)-\mathrm{W}(3)$ & $1.954(14)$ \\
\hline $\mathrm{O}(1)-\mathrm{W}(8)$ & $1.898(14)$ & $\mathrm{O}(40)-\mathrm{W}(3)$ & $1.872(14)$ \\
\hline $\mathrm{O}(1)-\mathrm{W}(9)$ & $1.940(14)$ & $\mathrm{O}(40)-\mathrm{W}(10)$ & $1.934(13)$ \\
\hline $\mathrm{O}(2)-\mathrm{P}(1)$ & $1.577(14)$ & $\mathrm{O}(41)-\mathrm{W}(17)$ & $1.896(16)$ \\
\hline $\mathrm{O}(2)-\mathrm{W}(7)$ & $2.363(14)$ & $\mathrm{O}(41)-\mathrm{W}(11)$ & $1.926(15)$ \\
\hline $\mathrm{O}(2)-\mathrm{W}(9)$ & $2.393(12)$ & $\mathrm{O}(42)-\mathrm{W}(11)$ & $1.881(15)$ \\
\hline $\mathrm{O}(2)-\mathrm{W}(8)$ & $2.397(13)$ & $\mathrm{O}(42)-\mathrm{W}(2)$ & $1.914(15)$ \\
\hline $\mathrm{O}(3)-\mathrm{P}(1)$ & $1.521(13)$ & $\mathrm{O}(43)-\mathrm{W}(12)$ & $1.896(15)$ \\
\hline $\mathrm{O}(3)-\mathrm{W}(5)$ & $2.336(13)$ & $\mathrm{O}(43)-\mathrm{W}(1)$ & $1.903(14)$ \\
\hline $\mathrm{O}(3)-\mathrm{W}(4)$ & $2.392(12)$ & $\mathrm{O}(44)-\mathrm{W}(13)$ & $1.889(13)$ \\
\hline $\mathrm{O}(4)-\mathrm{W}(9)$ & $1.891(15)$ & $\mathrm{O}(44)-\mathrm{W}(4)$ & $1.900(13)$ \\
\hline $\mathrm{O}(4)-\mathrm{W}(7)$ & $1.942(14)$ & $\mathrm{O}(45)-\mathrm{W}(6)$ & $1.869(14)$ \\
\hline $\mathrm{O}(3 \mathrm{~W})-\mathrm{Ag}(2)$ & $2.46(2)$ & $\mathrm{O}(45)-\mathrm{W}(18)$ & $1.924(14)$ \\
\hline $\mathrm{O}(5)-\mathrm{P}(1)$ & $1.527(14)$ & $\mathrm{O}(46)-\mathrm{W}(14)$ & $1.887(13)$ \\
\hline $\mathrm{O}(5)-\mathrm{W}(2)$ & $2.339(14)$ & $\mathrm{O}(46)-\mathrm{W}(18)$ & $1.907(13)$ \\
\hline $\mathrm{O}(5)-\mathrm{W}(1)$ & $2.393(13)$ & $\mathrm{O}(47)-\mathrm{W}(18)$ & $1.890(13)$ \\
\hline $\mathrm{O}(4 \mathrm{~W})-\mathrm{Ag}(3)$ & $2.56(3)$ & $\mathrm{O}(47)-\mathrm{W}(10)$ & $1.932(14)$ \\
\hline $\mathrm{O}(6)-\mathrm{W}(8)$ & $1.886(14)$ & $\mathrm{O}(48)-\mathrm{W}(10)$ & $1.689(14)$ \\
\hline $\mathrm{O}(6)-\mathrm{W}(6)$ & $1.952(14)$ & $\mathrm{O}(49)-\mathrm{W}(11)$ & $1.907(13)$ \\
\hline $\mathrm{O}(7)-\mathrm{P}(1)$ & $1.525(14)$ & $\mathrm{O}(49)-\mathrm{W}(10)$ & $1.907(14)$ \\
\hline $\mathrm{O}(7)-\mathrm{W}(3)$ & $2.348(13)$ & $\mathrm{O}(50)-\mathrm{W}(11)$ & $1.691(14)$ \\
\hline $\mathrm{O}(7)-\mathrm{W}(6)$ & $2.387(14)$ & $\mathrm{O}(51)-\mathrm{W}(13)$ & $1.700(13)$ \\
\hline $\mathrm{O}(8)-\mathrm{W}(7)$ & $1.901(14)$ & $\mathrm{O}(52)-\mathrm{W}(13)$ & $1.851(14)$ \\
\hline $\mathrm{O}(8)-\mathrm{W}(2)$ & $1.918(14)$ & $\mathrm{O}(52)-\mathrm{W}(12)$ & $1.929(13)$ \\
\hline $\mathrm{O}(9)-\mathrm{W}(6)$ & $1.898(13)$ & $\mathrm{O}(53)-\mathrm{W}(13)$ & $1.914(14)$ \\
\hline $\mathrm{O}(9)-\mathrm{W}(5)$ & $1.903(13)$ & $\mathrm{O}(53)-\mathrm{W}(14)$ & $1.932(13)$ \\
\hline $\mathrm{O}(10)-\mathrm{W}(5)$ & $1.915(13)$ & $\mathrm{O}(54)-\mathrm{W}(15)$ & $1.912(15)$ \\
\hline $\mathrm{O}(10)-\mathrm{W}(4)$ & $1.927(14)$ & $\mathrm{O}(54)-\mathrm{W}(14)$ & $1.935(16)$ \\
\hline $\mathrm{O}(11)-\mathrm{W}(6)$ & $1.916(13)$ & $\mathrm{O}(55)-\mathrm{W}(16)$ & $1.859(12)$ \\
\hline $\mathrm{O}(11)-\mathrm{W}(3)$ & $1.917(13)$ & $\mathrm{O}(55)-\mathrm{W}(13)$ & $1.971(13)$ \\
\hline $\mathrm{O}(12)-\mathrm{W}(1)$ & $1.881(14)$ & $\mathrm{O}(56)-\mathrm{W}(12)$ & $1.918(15)$ \\
\hline $\mathrm{O}(12)-\mathrm{W}(4)$ & $1.889(14)$ & $\mathrm{O}(56)-\mathrm{W}(16)$ & $1.920(16)$ \\
\hline $\mathrm{O}(13)-\mathrm{W}(2)$ & $1.898(13)$ & $\mathrm{O}(57)-\mathrm{W}(10)$ & $1.900(14)$ \\
\hline
\end{tabular}




\begin{tabular}{|c|c|c|c|}
\hline $\mathrm{O}(13)-\mathrm{W}(3)$ & $1.906(14)$ & $\mathrm{O}(57)-\mathrm{W}(17)$ & $1.947(13)$ \\
\hline $\mathrm{O}(14)-\mathrm{W}(7)$ & $1.703(16)$ & $\mathrm{O}(58)-\mathrm{W}(15)$ & $1.902(13)$ \\
\hline $\mathrm{O}(15)-\mathrm{W}(1)$ & $1.893(13)$ & $\mathrm{O}(58)-\mathrm{W}(18)$ & $1.922(13)$ \\
\hline $\mathrm{O}(15)-\mathrm{W}(2)$ & $1.944(13)$ & $\mathrm{O}(59)-\mathrm{P}(2)$ & $1.567(13)$ \\
\hline $\mathrm{O}(16)-\mathrm{W}(9)$ & $1.711(15)$ & $\mathrm{O}(59)-\mathrm{W}(15)$ & $2.385(14)$ \\
\hline $\mathrm{O}(17)-\mathrm{W}(14)$ & $1.874(14)$ & $\mathrm{O}(59)-\mathrm{W}(16)$ & $2.390(12)$ \\
\hline $\mathrm{O}(17)-\mathrm{W}(5)$ & $1.922(14)$ & $\mathrm{O}(59)-\mathrm{W}(17)$ & $2.395(12)$ \\
\hline $\mathrm{O}(18)-\mathrm{W}(9)$ & $1.906(14)$ & $\mathrm{O}(60)-\mathrm{P}(2)$ & $1.557(13)$ \\
\hline $\mathrm{O}(18)-\mathrm{W}(1)$ & $1.935(13)$ & $\mathrm{O}(60)-\mathrm{W}(18)$ & $2.338(14)$ \\
\hline $\mathrm{O}(19)-\mathrm{P}(2)$ & $1.518(12)$ & $\mathrm{O}(60)-\mathrm{W}(10)$ & $2.374(12)$ \\
\hline $\mathrm{O}(19)-\mathrm{W}(14)$ & $2.352(14)$ & $\mathrm{O}(61)-\mathrm{W}(15)$ & $1.918(14)$ \\
\hline $\mathrm{O}(19)-\mathrm{W}(13)$ & $2.378(12)$ & $\mathrm{O}(61)-\mathrm{W}(16)$ & $1.957(16)$ \\
\hline $\mathrm{O}(20)-\mathrm{W}(9)$ & $1.873(15)$ & $\mathrm{O}(62)-\mathrm{W}(17)$ & $1.881(14)$ \\
\hline $\mathrm{O}(20)-\mathrm{W}(4)$ & $1.923(14)$ & $\mathrm{O}(62)-\mathrm{W}(16)$ & $1.972(14)$ \\
\hline $\mathrm{O}(21)-\mathrm{P}(2)$ & $1.517(14)$ & $\operatorname{Ag}(1)-\mathrm{N}(11) \# 1$ & $2.16(2)$ \\
\hline $\mathrm{O}(21)-\mathrm{W}(12)$ & $2.375(12)$ & $\mathrm{Ag}(1)-\mathrm{O}(23) \# 2$ & $2.596(14)$ \\
\hline $\mathrm{O}(21)-\mathrm{W}(11)$ & $2.381(12)$ & $\operatorname{Ag}(1)-\operatorname{Ag}(4) \# 1$ & $2.817(3)$ \\
\hline $\mathrm{O}(22)-\mathrm{W}(8)$ & $1.907(15)$ & $\operatorname{Ag}(2)-\operatorname{Ag}(3)$ & $2.873(4)$ \\
\hline $\mathrm{O}(22)-\mathrm{W}(5)$ & $1.908(15)$ & $\operatorname{Ag}(4)-\operatorname{Ag}(1) \# 1$ & $2.817(3)$ \\
\hline $\mathrm{O}(23)-\mathrm{W}(18)$ & $1.686(16)$ & $\operatorname{Ag}(4)-\operatorname{Ag}(5)$ & $3.175(3)$ \\
\hline $\mathrm{O}(23)-\operatorname{Ag}(1) \# 2$ & $2.596(14)$ & $\operatorname{Ag}(5)-\mathrm{N}(23) \# 1$ & $2.530(18)$ \\
\hline $\mathrm{O}(24)-\mathrm{W}(8)$ & $1.897(18)$ & $\mathrm{Ag}(5)-\mathrm{O}(30) \# 4$ & $2.584(13)$ \\
\hline $\mathrm{O}(24)-\mathrm{W}(7)$ & $1.944(15)$ & $\mathrm{N}(23)-\operatorname{Ag}(5) \# 1$ & $2.530(18)$ \\
\hline $\mathrm{O}(25)-\mathrm{W}(8)$ & $1.716(13)$ & $\mathrm{N}(23)-\operatorname{Ag}(5) \# 1$ & $2.530(18)$ \\
\hline $\mathrm{O}(26)-\mathrm{W}(14)$ & $1.693(16)$ & $\mathrm{N}(23)-\operatorname{Ag}(5) \# 1$ & $2.530(18)$ \\
\hline $\mathrm{O}(27)-\mathrm{W}(12)$ & $1.716(13)$ & $\mathrm{N}(23)-\operatorname{Ag}(5) \# 1$ & $2.530(18)$ \\
\hline $\mathrm{O}(28)-\mathrm{W}(6)$ & $1.706(14)$ & $\mathrm{O}(30)-\mathrm{W}(5)$ & $1.706(14)$ \\
\hline $\mathrm{O}(29)-\mathrm{W}(11)$ & $1.894(14)$ & $\mathrm{O}(30)-\mathrm{Ag}(5) \# 3$ & $2.584(13)$ \\
\hline $\mathrm{O}(29)-\mathrm{W}(12)$ & $1.919(13)$ & $\mathrm{O}(31)-\mathrm{W}(15)$ & $1.727(18)$ \\
\hline $\mathrm{O}(3)-\mathrm{P}(1)-\mathrm{O}(7)$ & \begin{tabular}{l|l}
$113.1(8)$ \\
\end{tabular} & $\mathrm{O}(40)-\mathrm{W}(3)-\mathrm{O}(39)$ & $163.8(5)$ \\
\hline $\mathrm{O}(3)-\mathrm{P}(1)-\mathrm{O}(5)$ & $113.6(7)$ & $\mathrm{O}(13)-\mathrm{W}(3)-\mathrm{O}(39)$ & $83.9(6)$ \\
\hline $\mathrm{O}(7)-\mathrm{P}(1)-\mathrm{O}(5)$ & $110.8(7)$ & $\mathrm{O}(11)-\mathrm{W}(3)-\mathrm{O}(39)$ & $88.7(6)$ \\
\hline $\mathrm{O}(3)-\mathrm{P}(1)-\mathrm{O}(2)$ & $107.0(7)$ & $\mathrm{O}(37)-\mathrm{W}(3)-\mathrm{O}(7)$ & $171.8(5)$ \\
\hline $\mathrm{O}(7)-\mathrm{P}(1)-\mathrm{O}(2)$ & $106.2(7)$ & $\mathrm{O}(40)-\mathrm{W}(3)-\mathrm{O}(7)$ & $83.4(5)$ \\
\hline $\mathrm{O}(5)-\mathrm{P}(1)-\mathrm{O}(2)$ & $105.5(8)$ & $\mathrm{O}(13)-\mathrm{W}(3)-\mathrm{O}(7)$ & $85.7(5)$ \\
\hline $\mathrm{O}(21)-\mathrm{P}(2)-\mathrm{O}(19)$ & $112.2(7)$ & $\mathrm{O}(11)-\mathrm{W}(3)-\mathrm{O}(7)$ & $74.1(5)$ \\
\hline $\mathrm{O}(21)-\mathrm{P}(2)-\mathrm{O}(60)$ & $111.5(7)$ & $\mathrm{O}(39)-\mathrm{W}(3)-\mathrm{O}(7)$ & $81.1(5)$ \\
\hline $\mathrm{O}(19)-\mathrm{P}(2)-\mathrm{O}(60)$ & $112.3(7)$ & $\mathrm{O}(32)-\mathrm{W}(4)-\mathrm{O}(12)$ & 102.1(6) \\
\hline $\mathrm{O}(21)-\mathrm{P}(2)-\mathrm{O}(59)$ & $106.3(7)$ & $\mathrm{O}(32)-\mathrm{W}(4)-\mathrm{O}(44)$ & $98.1(6)$ \\
\hline $\mathrm{O}(19)-\mathrm{P}(2)-\mathrm{O}(59)$ & $106.5(8)$ & $\mathrm{O}(12)-\mathrm{W}(4)-\mathrm{O}(44)$ & $89.2(6)$ \\
\hline $\mathrm{O}(60)-\mathrm{P}(2)-\mathrm{O}(59)$ & $107.6(7)$ & $\mathrm{O}(32)-\mathrm{W}(4)-\mathrm{O}(20)$ & $99.8(6)$ \\
\hline $\mathrm{N}(11) \# 1-\mathrm{Ag}(1)-\mathrm{N}$ & $173.5(6)$ & $\mathrm{O}(12)-\mathrm{W}(4)-\mathrm{O}(20)$ & $86.4(6)$ \\
\hline $\mathrm{N}(13)-\operatorname{Ag}(2)-\mathrm{N}(7)$ & $131.4(7)$ & $\mathrm{O}(44)-\mathrm{W}(4)-\mathrm{O}(20)$ & $162.0(5)$ \\
\hline
\end{tabular}




\begin{tabular}{|c|c|c|c|}
\hline $\mathrm{N}(5)-\mathrm{Ag}(3)-\mathrm{N}(12)$ & $163.8(8)$ & $\mathrm{O}(32)-\mathrm{W}(4)-\mathrm{O}(10)$ & $101.1(6)$ \\
\hline $\mathrm{N}(8)-\operatorname{Ag}(4)-\mathrm{N}(6)$ & $170.2(7)$ & $\mathrm{O}(12)-\mathrm{W}(4)-\mathrm{O}(10)$ & $156.8(5)$ \\
\hline $\mathrm{N}(4)-\operatorname{Ag}(5)-\mathrm{N}(10)$ & $143.9(6)$ & $\mathrm{O}(44)-\mathrm{W}(4)-\mathrm{O}(10)$ & $87.4(5)$ \\
\hline $\mathrm{N}(4)-\mathrm{Ag}(5)-\mathrm{N}(23) \# 1$ & $106.7(7)$ & $\mathrm{O}(20)-\mathrm{W}(4)-\mathrm{O}(10)$ & $89.8(6)$ \\
\hline $\mathrm{N}(10)-\mathrm{Ag}(5)-\mathrm{N}(23) \# 1$ & $70.4(6)$ & $\mathrm{O}(32)-\mathrm{W}(4)-\mathrm{O}(3)$ & $173.6(6)$ \\
\hline $\mathrm{O}(33)-\mathrm{W}(1)-\mathrm{O}(12)$ & $102.2(6)$ & $\mathrm{O}(12)-\mathrm{W}(4)-\mathrm{O}(3)$ & $84.3(5)$ \\
\hline $\mathrm{O}(33)-\mathrm{W}(1)-\mathrm{O}(15)$ & $100.1(7)$ & $\mathrm{O}(44)-\mathrm{W}(4)-\mathrm{O}(3)$ & $82.1(5)$ \\
\hline $\mathrm{O}(12)-\mathrm{W}(1)-\mathrm{O}(15)$ & $157.7(6)$ & $\mathrm{O}(20)-\mathrm{W}(4)-\mathrm{O}(3)$ & $80.1(5)$ \\
\hline $\mathrm{O}(33)-\mathrm{W}(1)-\mathrm{O}(43)$ & $98.6(6)$ & $\mathrm{O}(10)-\mathrm{W}(4)-\mathrm{O}(3)$ & $72.5(5)$ \\
\hline $\mathrm{O}(12)-\mathrm{W}(1)-\mathrm{O}(43)$ & $87.0(6)$ & $\mathrm{O}(30)-\mathrm{W}(5)-\mathrm{O}(9)$ & $101.3(6)$ \\
\hline $\mathrm{O}(15)-\mathrm{W}(1)-\mathrm{O}(43)$ & $90.3(6)$ & $\mathrm{O}(30)-\mathrm{W}(5)-\mathrm{O}(22)$ & $98.9(6)$ \\
\hline $\mathrm{O}(33)-\mathrm{W}(1)-\mathrm{O}(18)$ & $97.3(6)$ & $\mathrm{O}(9)-\mathrm{W}(5)-\mathrm{O}(22)$ & $85.4(6)$ \\
\hline $\mathrm{O}(12)-\mathrm{W}(1)-\mathrm{O}(18)$ & $85.3(6)$ & $\mathrm{O}(30)-\mathrm{W}(5)-\mathrm{O}(10)$ & $100.1(6)$ \\
\hline $\mathrm{O}(15)-\mathrm{W}(1)-\mathrm{O}(18)$ & $91.3(6)$ & $\mathrm{O}(9)-\mathrm{W}(5)-\mathrm{O}(10)$ & $158.5(6)$ \\
\hline $\mathrm{O}(43)-\mathrm{W}(1)-\mathrm{O}(18)$ & $163.5(6)$ & $\mathrm{O}(22)-\mathrm{W}(5)-\mathrm{O}(10)$ & $92.2(6)$ \\
\hline $\mathrm{O}(33)-\mathrm{W}(1)-\mathrm{O}(5)$ & 173.1(6) & $\mathrm{O}(30)-\mathrm{W}(5)-\mathrm{O}(17)$ & $96.9(7)$ \\
\hline $\mathrm{O}(12)-\mathrm{W}(1)-\mathrm{O}(5)$ & $84.4(5)$ & $\mathrm{O}(9)-\mathrm{W}(5)-\mathrm{O}(17)$ & $87.7(6)$ \\
\hline $\mathrm{O}(15)-\mathrm{W}(1)-\mathrm{O}(5)$ & $73.3(5)$ & $\mathrm{O}(22)-\mathrm{W}(5)-\mathrm{O}(17)$ & $163.7(6)$ \\
\hline $\mathrm{O}(43)-\mathrm{W}(1)-\mathrm{O}(5)$ & $83.4(5)$ & $\mathrm{O}(10)-\mathrm{W}(5)-\mathrm{O}(17)$ & $88.8(5)$ \\
\hline $\mathrm{O}(18)-\mathrm{W}(1)-\mathrm{O}(5)$ & $81.4(5)$ & $\mathrm{O}(30)-\mathrm{W}(5)-\mathrm{O}(3)$ & $174.0(5)$ \\
\hline $\mathrm{O}(35)-\mathrm{W}(2)-\mathrm{O}(13)$ & $102.3(7)$ & $\mathrm{O}(9)-\mathrm{W}(5)-\mathrm{O}(3)$ & $84.5(5)$ \\
\hline $\mathrm{O}(35)-\mathrm{W}(2)-\mathrm{O}(42)$ & $98.3(7)$ & $\mathrm{O}(22)-\mathrm{W}(5)-\mathrm{O}(3)$ & $83.0(5)$ \\
\hline $\mathrm{O}(13)-\mathrm{W}(2)-\mathrm{O}(42)$ & $88.8(5)$ & $\mathrm{O}(10)-\mathrm{W}(5)-\mathrm{O}(3)$ & $74.0(5)$ \\
\hline $\mathrm{O}(35)-\mathrm{W}(2)-\mathrm{O}(8)$ & $99.0(7)$ & $\mathrm{O}(17)-\mathrm{W}(5)-\mathrm{O}(3)$ & $81.6(5)$ \\
\hline $\mathrm{O}(13)-\mathrm{W}(2)-\mathrm{O}(8)$ & $86.2(6)$ & $\mathrm{O}(28)-\mathrm{W}(6)-\mathrm{O}(45)$ & $99.8(7)$ \\
\hline $\mathrm{O}(42)-\mathrm{W}(2)-\mathrm{O}(8)$ & $162.6(6)$ & $\mathrm{O}(28)-\mathrm{W}(6)-\mathrm{O}(9)$ & $102.9(6)$ \\
\hline $\mathrm{O}(35)-\mathrm{W}(2)-\mathrm{O}(15)$ & $98.1(7)$ & $\mathrm{O}(45)-\mathrm{W}(6)-\mathrm{O}(9)$ & $90.4(6)$ \\
\hline $\mathrm{O}(13)-\mathrm{W}(2)-\mathrm{O}(15)$ & $159.6(6)$ & $\mathrm{O}(28)-\mathrm{W}(6)-\mathrm{O}(11)$ & $99.5(6)$ \\
\hline $\mathrm{O}(42)-\mathrm{W}(2)-\mathrm{O}(15)$ & $89.2(6)$ & $\mathrm{O}(45)-\mathrm{W}(6)-\mathrm{O}(11)$ & $90.1(6)$ \\
\hline $\mathrm{O}(8)-\mathrm{W}(2)-\mathrm{O}(15)$ & $89.7(6)$ & $\mathrm{O}(9)-\mathrm{W}(6)-\mathrm{O}(11)$ & $157.1(6)$ \\
\hline $\mathrm{O}(35)-\mathrm{W}(2)-\mathrm{O}(5)$ & $171.7(6)$ & $\mathrm{O}(28)-\mathrm{W}(6)-\mathrm{O}(6)$ & $96.8(6)$ \\
\hline $\mathrm{O}(13)-\mathrm{W}(2)-\mathrm{O}(5)$ & $85.9(5)$ & $\mathrm{O}(45)-\mathrm{W}(6)-\mathrm{O}(6)$ & $163.2(6)$ \\
\hline $\mathrm{O}(42)-\mathrm{W}(2)-\mathrm{O}(5)$ & $82.3(5)$ & $\mathrm{O}(9)-\mathrm{W}(6)-\mathrm{O}(6)$ & $83.8(6)$ \\
\hline $\mathrm{O}(8)-\mathrm{W}(2)-\mathrm{O}(5)$ & $80.7(5)$ & $\mathrm{O}(11)-\mathrm{W}(6)-\mathrm{O}(6)$ & $89.2(6)$ \\
\hline $\mathrm{O}(15)-\mathrm{W}(2)-\mathrm{O}(5)$ & $73.7(5)$ & $\mathrm{O}(28)-\mathrm{W}(6)-\mathrm{O}(7)$ & $172.5(6)$ \\
\hline $\mathrm{O}(37)-\mathrm{W}(3)-\mathrm{O}(40)$ & $100.4(6)$ & $\mathrm{O}(45)-\mathrm{W}(6)-\mathrm{O}(7)$ & $82.1(6)$ \\
\hline $\mathrm{O}(37)-\mathrm{W}(3)-\mathrm{O}(13)$ & $101.4(6)$ & $\mathrm{O}(9)-\mathrm{W}(6)-\mathrm{O}(7)$ & $84.2(5)$ \\
\hline $\mathrm{O}(40)-\mathrm{W}(3)-\mathrm{O}(13)$ & $90.2(6)$ & $\mathrm{O}(11)-\mathrm{W}(6)-\mathrm{O}(7)$ & $73.2(5)$ \\
\hline $\mathrm{O}(37)-\mathrm{W}(3)-\mathrm{O}(11)$ & $98.4(6)$ & $\mathrm{O}(6)-\mathrm{W}(6)-\mathrm{O}(7)$ & $81.6(5)$ \\
\hline $\mathrm{O}(40)-\mathrm{W}(3)-\mathrm{O}(11)$ & $91.7(6)$ & $\mathrm{O}(14)-\mathrm{W}(7)-\mathrm{O}(39)$ & $102.3(6)$ \\
\hline $\mathrm{O}(13)-\mathrm{W}(3)-\mathrm{O}(11)$ & $159.4(5)$ & $\mathrm{O}(14)-\mathrm{W}(7)-\mathrm{O}(8)$ & $103.6(7)$ \\
\hline $\mathrm{O}(37)-\mathrm{W}(3)-\mathrm{O}(39)$ & $95.6(6)$ & $\mathrm{O}(39)-\mathrm{W}(7)-\mathrm{O}(8)$ & $86.6(6)$ \\
\hline $\mathrm{O}(34)-\mathrm{W}(16)-\mathrm{O}(56)$ & $102.5(8)$ & $\mathrm{O}(14)-\mathrm{W}(7)-\mathrm{O}(4)$ & $101.5(7)$ \\
\hline
\end{tabular}




\begin{tabular}{|c|c|c|c|}
\hline $\mathrm{O}(55)-\mathrm{W}(16)-\mathrm{O}(56)$ & $86.9(6)$ & $\mathrm{O}(39)-\mathrm{W}(7)-\mathrm{O}(4)$ & $156.2(7)$ \\
\hline $\mathrm{O}(34)-\mathrm{W}(16)-\mathrm{O}(61)$ & $100.8(8)$ & $\mathrm{O}(8)-\mathrm{W}(7)-\mathrm{O}(4)$ & $88.4(6)$ \\
\hline $\mathrm{O}(55)-\mathrm{W}(16)-\mathrm{O}(61)$ & $88.9(6)$ & $\mathrm{O}(14)-\mathrm{W}(7)-\mathrm{O}(24)$ & $99.8(7)$ \\
\hline $\mathrm{O}(56)-\mathrm{W}(16)-\mathrm{O}(61)$ & $156.7(5)$ & $\mathrm{O}(39)-\mathrm{W}(7)-\mathrm{O}(24)$ & $89.2(6)$ \\
\hline $\mathrm{O}(34)-\mathrm{W}(16)-\mathrm{O}(62)$ & 101.1(6) & $\mathrm{O}(8)-\mathrm{W}(7)-\mathrm{O}(24)$ & $156.6(7)$ \\
\hline $\mathrm{O}(55)-\mathrm{W}(16)-\mathrm{O}(62)$ & $156.1(5)$ & $\mathrm{O}(4)-\mathrm{W}(7)-\mathrm{O}(24)$ & $86.3(6)$ \\
\hline $\mathrm{O}(56)-\mathrm{W}(16)-\mathrm{O}(62)$ & $87.0(6)$ & $\mathrm{O}(14)-\mathrm{W}(7)-\mathrm{O}(2)$ & $170.4(5)$ \\
\hline $\mathrm{O}(61)-\mathrm{W}(16)-\mathrm{O}(62)$ & $87.6(6)$ & $\mathrm{O}(39)-\mathrm{W}(7)-\mathrm{O}(2)$ & $84.0(5)$ \\
\hline $\mathrm{O}(34)-\mathrm{W}(16)-\mathrm{O}(59)$ & $171.8(6)$ & $\mathrm{O}(8)-\mathrm{W}(7)-\mathrm{O}(2)$ & $83.8(5)$ \\
\hline $\mathrm{O}(55)-\mathrm{W}(16)-\mathrm{O}(59)$ & $83.5(5)$ & $\mathrm{O}(4)-\mathrm{W}(7)-\mathrm{O}(2)$ & $72.3(6)$ \\
\hline $\mathrm{O}(56)-\mathrm{W}(16)-\mathrm{O}(59)$ & $82.9(5)$ & $\mathrm{O}(24)-\mathrm{W}(7)-\mathrm{O}(2)$ & $72.9(6)$ \\
\hline $\mathrm{O}(61)-\mathrm{W}(16)-\mathrm{O}(59)$ & $73.8(5)$ & $\mathrm{O}(25)-\mathrm{W}(8)-\mathrm{O}(6)$ & $103.1(6)$ \\
\hline $\mathrm{O}(62)-\mathrm{W}(16)-\mathrm{O}(59)$ & $72.8(5)$ & $\mathrm{O}(25)-\mathrm{W}(8)-\mathrm{O}(24)$ & $100.0(7)$ \\
\hline $\mathrm{O}(36)-\mathrm{W}(17)-\mathrm{O}(62)$ & $100.8(6)$ & $\mathrm{O}(6)-\mathrm{W}(8)-\mathrm{O}(24)$ & $89.5(7)$ \\
\hline $\mathrm{O}(36)-\mathrm{W}(17)-\mathrm{O}(41)$ & $102.3(8)$ & $\mathrm{O}(25)-\mathrm{W}(8)-\mathrm{O}(1)$ & $99.9(6)$ \\
\hline $\mathrm{O}(62)-\mathrm{W}(17)-\mathrm{O}(41)$ & $90.3(6)$ & $\mathrm{O}(6)-\mathrm{W}(8)-\mathrm{O}(1)$ & $157.0(6)$ \\
\hline $\mathrm{O}(36)-\mathrm{W}(17)-\mathrm{O}(38)$ & $100.9(8)$ & $\mathrm{O}(24)-\mathrm{W}(8)-\mathrm{O}(1)$ & $87.9(7)$ \\
\hline $\mathrm{O}(62)-\mathrm{W}(17)-\mathrm{O}(38)$ & $88.1(7)$ & $\mathrm{O}(25)-\mathrm{W}(8)-\mathrm{O}(22)$ & $103.3(7)$ \\
\hline $\mathrm{O}(41)-\mathrm{W}(17)-\mathrm{O}(38)$ & $156.6(6)$ & $\mathrm{O}(6)-\mathrm{W}(8)-\mathrm{O}(22)$ & $86.2(6)$ \\
\hline $\mathrm{O}(36)-\mathrm{W}(17)-\mathrm{O}(57)$ & $100.8(6)$ & $\mathrm{O}(24)-\mathrm{W}(8)-\mathrm{O}(22)$ & $156.7(5)$ \\
\hline $\mathrm{O}(62)-\mathrm{W}(17)-\mathrm{O}(57)$ & $158.4(5)$ & $\mathrm{O}(1)-\mathrm{W}(8)-\mathrm{O}(22)$ & $87.3(6)$ \\
\hline $\mathrm{O}(41)-\mathrm{W}(17)-\mathrm{O}(57)$ & $85.4(7)$ & $\mathrm{O}(25)-\mathrm{W}(8)-\mathrm{O}(2)$ & $170.0(7)$ \\
\hline $\mathrm{O}(38)-\mathrm{W}(17)-\mathrm{O}(57)$ & $87.5(6)$ & $\mathrm{O}(6)-\mathrm{W}(8)-\mathrm{O}(2)$ & $84.0(5)$ \\
\hline $\mathrm{O}(36)-\mathrm{W}(17)-\mathrm{O}(59)$ & $171.7(7)$ & $\mathrm{O}(24)-\mathrm{W}(8)-\mathrm{O}(2)$ & $72.8(5)$ \\
\hline $\mathrm{O}(62)-\mathrm{W}(17)-\mathrm{O}(59)$ & $74.1(5)$ & $\mathrm{O}(1)-\mathrm{W}(8)-\mathrm{O}(2)$ & $73.3(5)$ \\
\hline $\mathrm{O}(41)-\mathrm{W}(17)-\mathrm{O}(59)$ & $84.5(5)$ & $\mathrm{O}(22)-\mathrm{W}(8)-\mathrm{O}(2)$ & $83.9(5)$ \\
\hline $\mathrm{O}(38)-\mathrm{W}(17)-\mathrm{O}(59)$ & $72.6(5)$ & $\mathrm{O}(16)-\mathrm{W}(9)-\mathrm{O}(20)$ & $104.4(7)$ \\
\hline $\mathrm{O}(57)-\mathrm{W}(17)-\mathrm{O}(59)$ & $84.4(5)$ & $\mathrm{O}(16)-\mathrm{W}(9)-\mathrm{O}(4)$ & $99.5(7)$ \\
\hline $\mathrm{O}(23)-\mathrm{W}(18)-\mathrm{O}(47)$ & $99.2(7)$ & $\mathrm{O}(20)-\mathrm{W}(9)-\mathrm{O}(4)$ & $156.1(6)$ \\
\hline $\mathrm{O}(23)-\mathrm{W}(18)-\mathrm{O}(46)$ & 103.0(7) & $\mathrm{O}(16)-\mathrm{W}(9)-\mathrm{O}(18)$ & $102.0(6)$ \\
\hline $\mathrm{O}(47)-\mathrm{W}(18)-\mathrm{O}(46)$ & $157.8(6)$ & $\mathrm{O}(20)-\mathrm{W}(9)-\mathrm{O}(18)$ & $85.1(6)$ \\
\hline $\mathrm{O}(23)-\mathrm{W}(18)-\mathrm{O}(58)$ & $97.2(6)$ & $\mathrm{O}(4)-\mathrm{W}(9)-\mathrm{O}(18)$ & $89.0(6)$ \\
\hline $\mathrm{O}(47)-\mathrm{W}(18)-\mathrm{O}(58)$ & $91.6(5)$ & $\mathrm{O}(16)-\mathrm{W}(9)-\mathrm{O}(1)$ & $101.0(6)$ \\
\hline $\mathrm{O}(46)-\mathrm{W}(18)-\mathrm{O}(58)$ & $84.5(5)$ & $\mathrm{O}(20)-\mathrm{W}(9)-\mathrm{O}(1)$ & $89.2(6)$ \\
\hline $\mathrm{O}(23)-\mathrm{W}(18)-\mathrm{O}(45)$ & $99.2(7)$ & $\mathrm{O}(4)-\mathrm{W}(9)-\mathrm{O}(1)$ & $87.1(6)$ \\
\hline $\mathrm{O}(47)-\mathrm{W}(18)-\mathrm{O}(45)$ & $90.5(6)$ & $\mathrm{O}(18)-\mathrm{W}(9)-\mathrm{O}(1)$ & $157.0(6)$ \\
\hline $\mathrm{O}(46)-\mathrm{W}(18)-\mathrm{O}(45)$ & $87.2(6)$ & $\mathrm{O}(16)-\mathrm{W}(9)-\mathrm{O}(2)$ & $169.7(6)$ \\
\hline $\mathrm{O}(58)-\mathrm{W}(18)-\mathrm{O}(45)$ & $163.0(6)$ & $\mathrm{O}(20)-\mathrm{W}(9)-\mathrm{O}(2)$ & $83.9(5)$ \\
\hline $\mathrm{O}(23)-\mathrm{W}(18)-\mathrm{O}(60)$ & $173.2(6)$ & $\mathrm{O}(4)-\mathrm{W}(9)-\mathrm{O}(2)$ & $72.4(6)$ \\
\hline $\mathrm{O}(47)-\mathrm{W}(18)-\mathrm{O}(60)$ & $74.0(5)$ & $\mathrm{O}(18)-\mathrm{W}(9)-\mathrm{O}(2)$ & $84.5(5)$ \\
\hline $\mathrm{O}(46)-\mathrm{W}(18)-\mathrm{O}(60)$ & $83.8(5)$ & $\mathrm{O}(1)-\mathrm{W}(9)-\mathrm{O}(2)$ & $72.8(5)$ \\
\hline $\mathrm{O}(58)-\mathrm{W}(18)-\mathrm{O}(60)$ & $82.4(5)$ & $\mathrm{O}(48)-\mathrm{W}(10)-\mathrm{O}(57)$ & $98.5(6)$ \\
\hline $\mathrm{O}(45)-\mathrm{W}(18)-\mathrm{O}(60)$ & $82.0(5)$ & $\mathrm{O}(48)-\mathrm{W}(10)-\mathrm{O}(49)$ & $102.9(6)$ \\
\hline
\end{tabular}




\begin{tabular}{|c|c|c|c|}
\hline $\mathrm{O}(38)-\mathrm{W}(15)-\mathrm{O}(59)$ & $73.1(6)$ & $\mathrm{O}(57)-\mathrm{W}(10)-\mathrm{O}(49)$ & $89.2(6)$ \\
\hline $\mathrm{O}(54)-\mathrm{W}(15)-\mathrm{O}(59)$ & $83.7(5)$ & $\mathrm{O}(48)-\mathrm{W}(10)-\mathrm{O}(47)$ & $99.2(6)$ \\
\hline $\mathrm{O}(61)-\mathrm{W}(15)-\mathrm{O}(59)$ & $74.6(6)$ & $\mathrm{O}(57)-\mathrm{W}(10)-\mathrm{O}(47)$ & $89.5(6)$ \\
\hline $\mathrm{O}(34)-\mathrm{W}(16)-\mathrm{O}(55)$ & $102.8(6)$ & $\mathrm{O}(49)-\mathrm{W}(10)-\mathrm{O}(47)$ & $157.8(5)$ \\
\hline $\mathrm{O}(50)-\mathrm{W}(11)-\mathrm{O}(21)$ & $172.2(5)$ & $\mathrm{O}(48)-\mathrm{W}(10)-\mathrm{O}(40)$ & $98.0(6)$ \\
\hline $\mathrm{O}(42)-\mathrm{W}(11)-\mathrm{O}(21)$ & $82.4(5)$ & $\mathrm{O}(57)-\mathrm{W}(10)-\mathrm{O}(40)$ & $163.5(5)$ \\
\hline $\mathrm{O}(29)-\mathrm{W}(11)-\mathrm{O}(21)$ & $73.0(5)$ & $\mathrm{O}(49)-\mathrm{W}(10)-\mathrm{O}(40)$ & $86.6(6)$ \\
\hline $\mathrm{O}(49)-\mathrm{W}(11)-\mathrm{O}(21)$ & $85.1(5)$ & $\mathrm{O}(47)-\mathrm{W}(10)-\mathrm{O}(40)$ & $88.3(6)$ \\
\hline $\mathrm{O}(41)-\mathrm{W}(11)-\mathrm{O}(21)$ & $81.9(6)$ & $\mathrm{O}(48)-\mathrm{W}(10)-\mathrm{O}(60)$ & $171.6(6)$ \\
\hline $\mathrm{O}(27)-\mathrm{W}(12)-\mathrm{O}(43)$ & $99.5(6)$ & $\mathrm{O}(57)-\mathrm{W}(10)-\mathrm{O}(60)$ & $82.7(5)$ \\
\hline $\mathrm{O}(27)-\mathrm{W}(12)-\mathrm{O}(56)$ & $95.8(6)$ & $\mathrm{O}(49)-\mathrm{W}(10)-\mathrm{O}(60)$ & $85.4(5)$ \\
\hline $\mathrm{O}(43)-\mathrm{W}(12)-\mathrm{O}(56)$ & $164.6(5)$ & $\mathrm{O}(47)-\mathrm{W}(10)-\mathrm{O}(60)$ & $72.5(5)$ \\
\hline $\mathrm{O}(27)-\mathrm{W}(12)-\mathrm{O}(29)$ & $100.4(6)$ & $\mathrm{O}(40)-\mathrm{W}(10)-\mathrm{O}(60)$ & $81.1(5)$ \\
\hline $\mathrm{O}(43)-\mathrm{W}(12)-\mathrm{O}(29)$ & $89.7(6)$ & $\mathrm{O}(50)-\mathrm{W}(11)-\mathrm{O}(42)$ & $99.6(7)$ \\
\hline $\mathrm{O}(56)-\mathrm{W}(12)-\mathrm{O}(29)$ & $88.9(6)$ & $\mathrm{O}(50)-\mathrm{W}(11)-\mathrm{O}(29)$ & $99.5(6)$ \\
\hline $\mathrm{O}(27)-\mathrm{W}(12)-\mathrm{O}(52)$ & $102.2(6)$ & $\mathrm{O}(42)-\mathrm{W}(11)-\mathrm{O}(29)$ & $90.6(6)$ \\
\hline $\mathrm{O}(43)-\mathrm{W}(12)-\mathrm{O}(52)$ & $90.4(6)$ & $\mathrm{O}(50)-\mathrm{W}(11)-\mathrm{O}(49)$ & $102.5(6)$ \\
\hline $\mathrm{O}(56)-\mathrm{W}(12)-\mathrm{O}(52)$ & $85.1(6)$ & $\mathrm{O}(42)-\mathrm{W}(11)-\mathrm{O}(49)$ & $86.4(6)$ \\
\hline $\mathrm{O}(29)-\mathrm{W}(12)-\mathrm{O}(52)$ & $157.1(5)$ & $\mathrm{O}(29)-\mathrm{W}(11)-\mathrm{O}(49)$ & $158.1(5)$ \\
\hline $\mathrm{O}(27)-\mathrm{W}(12)-\mathrm{O}(21)$ & $172.6(5)$ & $\mathrm{O}(50)-\mathrm{W}(11)-\mathrm{O}(41)$ & $96.7(7)$ \\
\hline $\mathrm{O}(43)-\mathrm{W}(12)-\mathrm{O}(21)$ & $83.2(5)$ & $\mathrm{O}(42)-\mathrm{W}(11)-\mathrm{O}(41)$ & $163.4(6)$ \\
\hline $\mathrm{O}(56)-\mathrm{W}(12)-\mathrm{O}(21)$ & $81.8(5)$ & $\mathrm{O}(29)-\mathrm{W}(11)-\mathrm{O}(41)$ & $89.9(6)$ \\
\hline $\mathrm{O}(29)-\mathrm{W}(12)-\mathrm{O}(21)$ & $72.7(5)$ & $\mathrm{O}(49)-\mathrm{W}(11)-\mathrm{O}(41)$ & $86.9(6)$ \\
\hline $\mathrm{O}(52)-\mathrm{W}(12)-\mathrm{O}(21)$ & $84.6(5)$ & $\mathrm{O}(26)-\mathrm{W}(14)-\mathrm{O}(53)$ & $99.8(6)$ \\
\hline $\mathrm{O}(51)-\mathrm{W}(13)-\mathrm{O}(52)$ & $102.5(6)$ & $\mathrm{O}(17)-\mathrm{W}(14)-\mathrm{O}(53)$ & $91.0(5)$ \\
\hline $\mathrm{O}(51)-\mathrm{W}(13)-\mathrm{O}(44)$ & $99.3(6)$ & $\mathrm{O}(46)-\mathrm{W}(14)-\mathrm{O}(53)$ & $157.4(5)$ \\
\hline $\mathrm{O}(52)-\mathrm{W}(13)-\mathrm{O}(44)$ & $89.2(6)$ & $\mathrm{O}(26)-\mathrm{W}(14)-\mathrm{O}(54)$ & $98.7(7)$ \\
\hline $\mathrm{O}(51)-\mathrm{W}(13)-\mathrm{O}(53)$ & $100.0(6)$ & $\mathrm{O}(17)-\mathrm{W}(14)-\mathrm{O}(54)$ & $162.8(6)$ \\
\hline $\mathrm{O}(52)-\mathrm{W}(13)-\mathrm{O}(53)$ & $156.8(5)$ & $\mathrm{O}(46)-\mathrm{W}(14)-\mathrm{O}(54)$ & $87.1(6)$ \\
\hline $\mathrm{O}(44)-\mathrm{W}(13)-\mathrm{O}(53)$ & $92.6(5)$ & $\mathrm{O}(53)-\mathrm{W}(14)-\mathrm{O}(54)$ & $86.0(5)$ \\
\hline $\mathrm{O}(51)-\mathrm{W}(13)-\mathrm{O}(55)$ & $97.6(6)$ & $\mathrm{O}(26)-\mathrm{W}(14)-\mathrm{O}(19)$ & $173.0(6)$ \\
\hline $\mathrm{O}(52)-\mathrm{W}(13)-\mathrm{O}(55)$ & $83.8(6)$ & $\mathrm{O}(17)-\mathrm{W}(14)-\mathrm{O}(19)$ & $81.8(5)$ \\
\hline $\mathrm{O}(44)-\mathrm{W}(13)-\mathrm{O}(55)$ & $162.8(5)$ & $\mathrm{O}(46)-\mathrm{W}(14)-\mathrm{O}(19)$ & $84.6(5)$ \\
\hline $\mathrm{O}(53)-\mathrm{W}(13)-\mathrm{O}(55)$ & $87.9(6)$ & $\mathrm{O}(53)-\mathrm{W}(14)-\mathrm{O}(19)$ & $73.1(5)$ \\
\hline $\mathrm{O}(51)-\mathrm{W}(13)-\mathrm{O}(19)$ & $172.7(6)$ & $\mathrm{O}(54)-\mathrm{W}(14)-\mathrm{O}(19)$ & $81.2(5)$ \\
\hline $\mathrm{O}(52)-\mathrm{W}(13)-\mathrm{O}(19)$ & $84.5(5)$ & $\mathrm{O}(31)-\mathrm{W}(15)-\mathrm{O}(58)$ & $101.3(7)$ \\
\hline $\mathrm{O}(44)-\mathrm{W}(13)-\mathrm{O}(19)$ & $82.8(5)$ & $\mathrm{O}(31)-\mathrm{W}(15)-\mathrm{O}(38)$ & $99.6(7)$ \\
\hline $\mathrm{O}(53)-\mathrm{W}(13)-\mathrm{O}(19)$ & $72.8(5)$ & $\mathrm{O}(58)-\mathrm{W}(15)-\mathrm{O}(38)$ & $89.5(6)$ \\
\hline $\mathrm{O}(55)-\mathrm{W}(13)-\mathrm{O}(19)$ & $80.8(5)$ & $\mathrm{O}(31)-\mathrm{W}(15)-\mathrm{O}(54)$ & $103.7(7)$ \\
\hline $\mathrm{O}(26)-\mathrm{W}(14)-\mathrm{O}(17)$ & $98.5(6)$ & $\mathrm{O}(58)-\mathrm{W}(15)-\mathrm{O}(54)$ & $85.7(5)$ \\
\hline $\mathrm{O}(26)-\mathrm{W}(14)-\mathrm{O}(46)$ & $102.5(6)$ & $\mathrm{O}(38)-\mathrm{W}(15)-\mathrm{O}(54)$ & $156.7(7)$ \\
\hline $\mathrm{O}(17)-\mathrm{W}(14)-\mathrm{O}(46)$ & $89.4(6)$ & $\mathrm{O}(31)-\mathrm{W}(15)-\mathrm{O}(61)$ & $99.9(7)$ \\
\hline $\mathrm{O}(54)-\mathrm{W}(15)-\mathrm{O}(61)$ & $88.6(6)$ & $\mathrm{O}(58)-\mathrm{W}(15)-\mathrm{O}(61)$ & $158.9(6)$ \\
\hline
\end{tabular}




\begin{tabular}{|l|l|l|l|}
\hline $\mathrm{O}(31)-\mathrm{W}(15)-\mathrm{O}(59)$ & $170.8(6)$ & $\mathrm{O}(38)-\mathrm{W}(15)-\mathrm{O}(61)$ & $87.7(6)$ \\
\hline
\end{tabular}

Table S3. Selected Bond Lengths $(\AA)$ and Bond Angles $\left({ }^{\circ}\right)$ for Compound 2.

\begin{tabular}{|c|c|c|c|}
\hline $\operatorname{Ag}(1)-\mathrm{N}(10)$ & $2.14(2)$ & $\mathrm{O}(27)-\mathrm{W}(13)$ & $2.383(15)$ \\
\hline $\operatorname{Ag}(1)-\mathrm{N}(9)$ & $2.26(2)$ & $\mathrm{O}(28)-\mathrm{W}(1)$ & $1.877(15)$ \\
\hline $\operatorname{Ag}(1)-O(1 W)$ & $2.39(2)$ & $\mathrm{O}(28)-\mathrm{W}(18)$ & $1.902(15)$ \\
\hline $\operatorname{Ag}(1)-\operatorname{Ag}(5)$ & $2.953(5)$ & $\mathrm{O}(29)-\mathrm{W}(18)$ & $1.872(17)$ \\
\hline $\operatorname{Ag}(2)-\mathrm{N}(1)$ & $2.17(2)$ & $\mathrm{O}(29)-\mathrm{W}(13)$ & $1.901(17)$ \\
\hline $\operatorname{Ag}(2)-\mathrm{N}(5)$ & $2.20(2)$ & $\mathrm{O}(30)-\mathrm{W}(18)$ & $1.693(17)$ \\
\hline $\operatorname{Ag}(2)-\operatorname{Ag}(6)$ & $2.807(6)$ & $\mathrm{O}(31)-\mathrm{W}(12)$ & $1.894(16)$ \\
\hline $\operatorname{Ag}(2)-\operatorname{Ag}(4)$ & $3.216(3)$ & $\mathrm{O}(31)-\mathrm{W}(1)$ & $1.895(15)$ \\
\hline $\operatorname{Ag}(3)-\mathrm{N}(8) \# 1$ & $2.292(19)$ & $\mathrm{O}(32)-\mathrm{W}(16)$ & $1.894(17)$ \\
\hline $\operatorname{Ag}(3)-\mathrm{N}(8)$ & $2.292(19)$ & $\mathrm{O}(32)-\mathrm{W}(13)$ & $1.914(16)$ \\
\hline $\operatorname{Ag}(3)-N(20) \# 1$ & $2.55(2)$ & $\mathrm{O}(33)-\mathrm{W}(16)$ & $1.903(17)$ \\
\hline $\operatorname{Ag}(3)-\mathrm{N}(20)$ & $2.55(2)$ & $\mathrm{O}(33)-\mathrm{W}(9)$ & $1.905(17)$ \\
\hline $\mathrm{Ag}(4)-\mathrm{N}(3) \# 2$ & $2.20(2)$ & $\mathrm{O}(34)-\mathrm{W}(12)$ & $1.867(15)$ \\
\hline $\operatorname{Ag}(4)-\mathrm{N}(2)$ & $2.24(2)$ & $\mathrm{O}(34)-\mathrm{W}(8)$ & $1.911(15)$ \\
\hline $\operatorname{Ag}(4)-O(16)$ & $2.579(18)$ & $\mathrm{O}(35)-\mathrm{W}(8)$ & $1.880(15)$ \\
\hline $\operatorname{Ag}(5)-\mathrm{N}(13)$ & $1.97(4)$ & $\mathrm{O}(35)-\mathrm{W}(11)$ & $1.940(16)$ \\
\hline $\operatorname{Ag}(5)-\mathrm{N}(6)$ & $2.13(2)$ & $\mathrm{O}(36)-\mathrm{P}(2)$ & $1.522(16)$ \\
\hline $\operatorname{Ag}(6)-\mathrm{N}(14)$ & $2.014(13)$ & $\mathrm{O}(36)-\mathrm{W}(11)$ & $2.357(15)$ \\
\hline $\operatorname{Ag}(6)-N(21)$ & $2.41(2)$ & $\mathrm{O}(36)-\mathrm{W}(8)$ & $2.358(16)$ \\
\hline $\operatorname{Ag}(6)-\mathrm{N}(23)$ & $2.56(3)$ & $\mathrm{O}(37)-\mathrm{W}(9)$ & $1.707(16)$ \\
\hline $\operatorname{Ag}(7)-O(7 W)$ & $2.02(3)$ & $\mathrm{O}(38)-\mathrm{W}(11)$ & $1.873(17)$ \\
\hline $\operatorname{Ag}(7)-N(4)$ & $2.19(2)$ & $\mathrm{O}(38)-\mathrm{W}(4)$ & $1.906(17)$ \\
\hline $\mathrm{O}(26)-\mathrm{W}(1)$ & $2.366(16)$ & $\mathrm{O}(39)-\mathrm{W}(17)$ & $1.905(17)$ \\
\hline $\mathrm{O}(26)-\mathrm{W}(18)$ & $2.375(15)$ & $\mathrm{O}(39)-\mathrm{W}(8)$ & $1.934(17)$ \\
\hline $\mathrm{O}(27)-\mathrm{P}(1)$ & $1.517(15)$ & $\mathrm{O}(40)-\mathrm{W}(11)$ & $1.910(17)$ \\
\hline $\mathrm{O}(27)-\mathrm{W}(16)$ & $2.351(14)$ & $\mathrm{O}(40)-\mathrm{W}(5)$ & $1.913(17)$ \\
\hline $\mathrm{O}(20)-\mathrm{W}(15)$ & $1.869(17)$ & $\mathrm{O}(41)-\mathrm{W}(17)$ & $1.913(18)$ \\
\hline $\mathrm{O}(20)-\mathrm{W}(10)$ & $1.955(16)$ & $\mathrm{O}(41)-\mathrm{W}(5)$ & $1.951(18)$ \\
\hline $\mathrm{O}(21)-\mathrm{W}(15)$ & $1.708(16)$ & $\mathrm{O}(42)-\mathrm{W}(5)$ & $1.665(16)$ \\
\hline $\mathrm{O}(22)-\mathrm{W}(15)$ & $1.881(17)$ & $\mathrm{O}(43)-\mathrm{W}(5)$ & $1.921(18)$ \\
\hline $\mathrm{O}(1)-\mathrm{W}(12)$ & $1.683(17)$ & $\mathrm{O}(43)-\mathrm{W}(4)$ & $1.925(17)$ \\
\hline $\mathrm{O}(2)-\mathrm{W}(3)$ & $1.884(15)$ & $\mathrm{O}(44)-\mathrm{W}(17)$ & $1.860(17)$ \\
\hline $\mathrm{O}(2)-\mathrm{W}(12)$ & $1.912(15)$ & $\mathrm{O}(44)-\mathrm{W}(12)$ & $1.956(17)$ \\
\hline $\mathrm{O}(3)-\mathrm{W}(18)$ & $1.848(15)$ & $\mathrm{O}(45)-\mathrm{W}(17)$ & $1.892(17)$ \\
\hline $\mathrm{O}(3)-\mathrm{W}(3)$ & $1.956(15)$ & $\mathrm{O}(45)-\mathrm{W}(7)$ & $1.952(16)$ \\
\hline $\mathrm{O}(4)-\mathrm{W}(3)$ & $1.718(16)$ & $\mathrm{O}(46)-\mathrm{W}(7)$ & $1.713(17)$ \\
\hline $\mathrm{O}(5)-\mathrm{P}(2)$ & $1.528(16)$ & $\mathrm{O}(47)-\mathrm{W}(4)$ & $1.890(16)$ \\
\hline $\mathrm{O}(5)-\mathrm{W}(12)$ & $2.344(15)$ & $\mathrm{O}(47)-\mathrm{W}(16)$ & $1.911(16)$ \\
\hline $\mathrm{O}(5)-\mathrm{W}(3)$ & $2.354(15)$ & $\mathrm{O}(48)-\mathrm{W}(13)$ & $1.895(16)$ \\
\hline $\mathrm{O}(6)-\mathrm{W}(14)$ & $1.880(15)$ & $\mathrm{O}(48)-\mathrm{W}(14)$ & $1.902(16)$ \\
\hline
\end{tabular}




\begin{tabular}{|c|c|c|c|}
\hline $\mathrm{O}(6)-\mathrm{W}(3)$ & $1.898(16)$ & $\mathrm{O}(49)-\mathrm{W}(17)$ & $1.681(18)$ \\
\hline $\mathrm{O}(7)-\mathrm{P}(2)$ & $1.496(16)$ & $\mathrm{O}(50)-\mathrm{W}(2)$ & $1.914(18)$ \\
\hline $\mathrm{O}(7)-\mathrm{W}(14)$ & $2.378(16)$ & $\mathrm{O}(50)-\mathrm{W}(10)$ & $1.915(18)$ \\
\hline $\mathrm{O}(7)-\mathrm{W}(4)$ & $2.387(15)$ & $\mathrm{O}(51)-\mathrm{W}(4)$ & $1.882(15)$ \\
\hline $\mathrm{O}(8)-\mathrm{P}(2)$ & $1.574(17)$ & $\mathrm{O}(51)-\mathrm{W}(14)$ & $1.925(15)$ \\
\hline $\mathrm{O}(8)-\mathrm{W}(17)$ & $2.353(16)$ & $\mathrm{O}(52)-\mathrm{W}(4)$ & $1.704(16)$ \\
\hline $\mathrm{O}(8)-\mathrm{W}(5)$ & $2.372(18)$ & $\mathrm{O}(53)-\mathrm{W}(1)$ & $1.900(15)$ \\
\hline $\mathrm{O}(8)-\mathrm{W}(7)$ & $2.398(17)$ & $\mathrm{O}(53)-\mathrm{W}(2)$ & $1.910(16)$ \\
\hline $\mathrm{O}(9)-\mathrm{W}(7)$ & $1.889(17)$ & $\mathrm{O}(54)-\mathrm{W}(2)$ & $1.906(15)$ \\
\hline $\mathrm{O}(9)-\mathrm{W}(5)$ & $1.925(16)$ & $\mathrm{O}(54)-\mathrm{W}(15)$ & $1.952(16)$ \\
\hline $\mathrm{O}(10)-\mathrm{W}(1)$ & $1.692(16)$ & $\mathrm{O}(55)-\mathrm{W}(13)$ & $1.743(18)$ \\
\hline $\mathrm{O}(11)-\mathrm{W}(6)$ & $1.883(16)$ & $\mathrm{O}(56)-\mathrm{W}(7)$ & $1.863(16)$ \\
\hline $\mathrm{O}(11)-\mathrm{W}(1)$ & $1.895(17)$ & $\mathrm{O}(56)-\mathrm{W}(14)$ & $1.974(16)$ \\
\hline $\mathrm{O}(12)-\mathrm{W}(10)$ & $1.665(18)$ & $\mathrm{O}(57)-\mathrm{W}(6)$ & $1.722(18)$ \\
\hline $\mathrm{O}(13)-\mathrm{W}(8)$ & $1.894(15)$ & $\mathrm{O}(58)-\mathrm{W}(9)$ & $1.906(17)$ \\
\hline $\mathrm{O}(13)-\mathrm{W}(6)$ & $1.897(15)$ & $\mathrm{O}(58)-\mathrm{W}(6)$ & $1.937(15)$ \\
\hline $\mathrm{O}(14)-\mathrm{W}(11)$ & $1.847(16)$ & $\mathrm{O}(59)-\mathrm{W}(7)$ & $1.903(17)$ \\
\hline $\mathrm{O}(14)-\mathrm{W}(9)$ & $1.941(16)$ & $\mathrm{O}(59)-\mathrm{W}(3)$ & $1.912(16)$ \\
\hline $\mathrm{O}(15)-\mathrm{W}(2)$ & $1.699(17)$ & $\mathrm{O}(60)-\mathrm{W}(14)$ & $1.692(16)$ \\
\hline $\mathrm{O}(16)-\mathrm{W}(16)$ & $1.693(17)$ & $\mathrm{O}(61)-\mathrm{W}(11)$ & $1.713(16)$ \\
\hline $\mathrm{O}(17)-\mathrm{P}(1)$ & $1.574(15)$ & $\mathrm{O}(62)-\mathrm{W}(8)$ & $1.708(17)$ \\
\hline $\mathrm{O}(17)-\mathrm{W}(15)$ & $2.343(14)$ & $\mathrm{O}(22)-\mathrm{W}(9)$ & $1.921(17)$ \\
\hline $\mathrm{O}(17)-\mathrm{W}(2)$ & $2.388(15)$ & $\mathrm{O}(23)-\mathrm{W}(2)$ & $1.898(17)$ \\
\hline $\mathrm{O}(17)-\mathrm{W}(10)$ & $2.397(15)$ & $\mathrm{O}(23)-\mathrm{W}(6)$ & $1.904(17)$ \\
\hline $\mathrm{O}(18)-\mathrm{W}(15)$ & $1.881(15)$ & $\mathrm{O}(24)-\mathrm{W}(10)$ & $1.895(16)$ \\
\hline $\mathrm{O}(18)-\mathrm{W}(16)$ & $1.934(15)$ & $\mathrm{O}(24)-\mathrm{W}(13)$ & $1.916(16)$ \\
\hline $\mathrm{O}(19)-\mathrm{W}(10)$ & $1.855(15)$ & $\mathrm{O}(25)-\mathrm{P}(1)$ & $1.568(17)$ \\
\hline $\mathrm{O}(19)-\mathrm{W}(18)$ & $1.987(15)$ & $\mathrm{O}(25)-\mathrm{W}(6)$ & $2.328(17)$ \\
\hline $\mathrm{O}(26)-\mathrm{P}(1)$ & $1.524(15)$ & $\mathrm{O}(25)-\mathrm{W}(9)$ & $2.347(15)$ \\
\hline $\mathrm{N}(10)-\operatorname{Ag}(1)-\mathrm{N}(9)$ & $153.5(8)$ & $\mathrm{O}(38)-\mathrm{W}(11)-\mathrm{O}(40)$ & $85.3(7)$ \\
\hline $\mathrm{N}(1)-\mathrm{Ag}(2)-\mathrm{N}(5)$ & $170.7(9)$ & $\mathrm{O}(61)-\mathrm{W}(11)-\mathrm{O}(35)$ & $99.1(7)$ \\
\hline $\mathrm{N}(8) \# 1-\mathrm{Ag}(3)-\mathrm{N}(8)$ & $135.6(10)$ & $\mathrm{O}(14)-\mathrm{W}(11)-\mathrm{O}(35)$ & $90.4(7)$ \\
\hline $\mathrm{N}(8) \# 1-\mathrm{Ag}(3)-\mathrm{N}(20) \# 1$ & $73.8(7)$ & $\mathrm{O}(38)-\mathrm{W}(11)-\mathrm{O}(35)$ & $157.5(7)$ \\
\hline $\mathrm{N}(8)-\operatorname{Ag}(3)-\mathrm{N}(20) \# 1$ & $143.4(7)$ & $\mathrm{O}(40)-\mathrm{W}(11)-\mathrm{O}(35)$ & $89.1(7)$ \\
\hline $\mathrm{N}(8) \# 1-\operatorname{Ag}(3)-\mathrm{N}(20)$ & $143.4(7)$ & $\mathrm{O}(61)-\mathrm{W}(11)-\mathrm{O}(36)$ & $171.6(7)$ \\
\hline $\mathrm{N}(8)-\operatorname{Ag}(3)-\mathrm{N}(20)$ & $73.8(7)$ & $\mathrm{O}(14)-\mathrm{W}(11)-\mathrm{O}(36)$ & $84.4(6)$ \\
\hline $\mathrm{N}(20) \# 1-\operatorname{Ag}(3)-\mathrm{N}(20)$ & $92.0(10)$ & $\mathrm{O}(38)-\mathrm{W}(11)-\mathrm{O}(36)$ & $84.6(6)$ \\
\hline $\mathrm{N}(3) \# 2-\mathrm{Ag}(4)-\mathrm{N}(2)$ & $145.4(8)$ & $\mathrm{O}(40)-\mathrm{W}(11)-\mathrm{O}(36)$ & $80.8(6)$ \\
\hline $\mathrm{N}(001)-\operatorname{Ag}(5)-\mathrm{N}(13)$ & $41.1(12)$ & $\mathrm{O}(35)-\mathrm{W}(11)-\mathrm{O}(36)$ & $73.0(6)$ \\
\hline $\mathrm{N}(001)-\mathrm{Ag}(5)-\mathrm{N}(6)$ & $145.0(17)$ & $\mathrm{O}(1)-\mathrm{W}(12)-\mathrm{O}(34)$ & $102.4(8)$ \\
\hline $\mathrm{N}(13)-\operatorname{Ag}(5)-\mathrm{N}(6)$ & $168.9(12)$ & $\mathrm{O}(1)-\mathrm{W}(12)-\mathrm{O}(31)$ & $99.6(8)$ \\
\hline $\mathrm{N}(14)-\mathrm{Ag}(6)-\mathrm{N}(21)$ & $97.5(11)$ & $\mathrm{O}(34)-\mathrm{W}(12)-\mathrm{O}(31)$ & $90.8(6)$ \\
\hline
\end{tabular}




\begin{tabular}{|c|c|c|c|}
\hline $\mathrm{N}(14)-\operatorname{Ag}(6)-\mathrm{N}(23)$ & $112.3(13)$ & $\mathrm{O}(1)-\mathrm{W}(12)-\mathrm{O}(2)$ & $100.1(7)$ \\
\hline $\mathrm{N}(21)-\mathrm{Ag}(6)-\mathrm{N}(23)$ & $142.0(10)$ & $\mathrm{O}(34)-\mathrm{W}(12)-\mathrm{O}(2)$ & $157.4(6)$ \\
\hline $\mathrm{O}(27)-\mathrm{P}(1)-\mathrm{O}(26)$ & $113.2(9)$ & $\mathrm{O}(31)-\mathrm{W}(12)-\mathrm{O}(2)$ & $88.0(6)$ \\
\hline $\mathrm{O}(27)-\mathrm{P}(1)-\mathrm{O}(25)$ & 111.1(9) & $\mathrm{O}(1)-\mathrm{W}(12)-\mathrm{O}(44)$ & $96.9(8)$ \\
\hline $\mathrm{O}(26)-\mathrm{P}(1)-\mathrm{O}(25)$ & $110.9(9)$ & $\mathrm{O}(34)-\mathrm{W}(12)-\mathrm{O}(44)$ & $85.7(6)$ \\
\hline $\mathrm{O}(27)-\mathrm{P}(1)-\mathrm{O}(17)$ & 108.1(9) & $\mathrm{O}(31)-\mathrm{W}(12)-\mathrm{O}(44)$ & $163.5(7)$ \\
\hline $\mathrm{O}(26)-\mathrm{P}(1)-\mathrm{O}(17)$ & $107.2(8)$ & $\mathrm{O}(2)-\mathrm{W}(12)-\mathrm{O}(44)$ & $89.1(6)$ \\
\hline $\mathrm{O}(25)-\mathrm{P}(1)-\mathrm{O}(17)$ & $105.9(9)$ & $\mathrm{O}(1)-\mathrm{W}(12)-\mathrm{O}(5)$ & $171.7(7)$ \\
\hline $\mathrm{O}(7)-\mathrm{P}(2)-\mathrm{O}(36)$ & $112.9(9)$ & $\mathrm{O}(34)-\mathrm{W}(12)-\mathrm{O}(5)$ & $85.2(6)$ \\
\hline $\mathrm{O}(7)-\mathrm{P}(2)-\mathrm{O}(5)$ & $111.8(9)$ & $\mathrm{O}(31)-\mathrm{W}(12)-\mathrm{O}(5)$ & $83.4(6)$ \\
\hline $\mathrm{O}(36)-\mathrm{P}(2)-\mathrm{O}(5)$ & $111.6(9)$ & $\mathrm{O}(2)-\mathrm{W}(12)-\mathrm{O}(5)$ & $72.3(6)$ \\
\hline $\mathrm{O}(7)-\mathrm{P}(2)-\mathrm{O}(8)$ & $108.0(9)$ & $\mathrm{O}(44)-\mathrm{W}(12)-\mathrm{O}(5)$ & $80.2(6)$ \\
\hline $\mathrm{O}(36)-\mathrm{P}(2)-\mathrm{O}(8)$ & 106.1(9) & $\mathrm{O}(55)-\mathrm{W}(13)-\mathrm{O}(48)$ & $98.9(8)$ \\
\hline $\mathrm{O}(5)-\mathrm{P}(2)-\mathrm{O}(8)$ & $106.0(10)$ & $\mathrm{O}(55)-\mathrm{W}(13)-\mathrm{O}(29)$ & $101.5(8)$ \\
\hline $\mathrm{O}(10)-\mathrm{W}(1)-\mathrm{O}(28)$ & $102.4(8)$ & $\mathrm{O}(48)-\mathrm{W}(13)-\mathrm{O}(29)$ & $87.4(7)$ \\
\hline $\mathrm{O}(10)-\mathrm{W}(1)-\mathrm{O}(31)$ & $99.9(7)$ & $\mathrm{O}(55)-\mathrm{W}(13)-\mathrm{O}(32)$ & $101.4(8)$ \\
\hline $\mathrm{O}(28)-\mathrm{W}(1)-\mathrm{O}(31)$ & $88.4(7)$ & $\mathrm{O}(48)-\mathrm{W}(13)-\mathrm{O}(32)$ & $89.5(7)$ \\
\hline $\mathrm{O}(10)-\mathrm{W}(1)-\mathrm{O}(11)$ & $102.9(8)$ & $\mathrm{O}(29)-\mathrm{W}(13)-\mathrm{O}(32)$ & $157.1(7)$ \\
\hline $\mathrm{O}(28)-\mathrm{W}(1)-\mathrm{O}(11)$ & $154.7(7)$ & $\mathrm{O}(55)-\mathrm{W}(13)-\mathrm{O}(24)$ & $97.7(8)$ \\
\hline $\mathrm{O}(31)-\mathrm{W}(1)-\mathrm{O}(11)$ & $87.9(7)$ & $\mathrm{O}(48)-\mathrm{W}(13)-\mathrm{O}(24)$ & $163.0(7)$ \\
\hline $\mathrm{O}(10)-\mathrm{W}(1)-\mathrm{O}(53)$ & $95.7(7)$ & $\mathrm{O}(29)-\mathrm{W}(13)-\mathrm{O}(24)$ & $85.7(7)$ \\
\hline $\mathrm{O}(28)-\mathrm{W}(1)-\mathrm{O}(53)$ & $90.3(7)$ & $\mathrm{O}(32)-\mathrm{W}(13)-\mathrm{O}(24)$ & $90.9(7)$ \\
\hline $\mathrm{O}(31)-\mathrm{W}(1)-\mathrm{O}(53)$ & $164.2(7)$ & $\mathrm{O}(55)-\mathrm{W}(13)-\mathrm{O}(27)$ & $173.8(7)$ \\
\hline $\mathrm{O}(11)-\mathrm{W}(1)-\mathrm{O}(53)$ & $86.6(7)$ & $\mathrm{O}(48)-\mathrm{W}(13)-\mathrm{O}(27)$ & $82.8(6)$ \\
\hline $\mathrm{O}(10)-\mathrm{W}(1)-\mathrm{O}(26)$ & $174.3(7)$ & $\mathrm{O}(29)-\mathrm{W}(13)-\mathrm{O}(27)$ & $84.4(6)$ \\
\hline $\mathrm{O}(28)-\mathrm{W}(1)-\mathrm{O}(26)$ & $72.0(6)$ & $\mathrm{O}(32)-\mathrm{W}(13)-\mathrm{O}(27)$ & $72.7(6)$ \\
\hline $\mathrm{O}(31)-\mathrm{W}(1)-\mathrm{O}(26)$ & $81.3(6)$ & $\mathrm{O}(24)-\mathrm{W}(13)-\mathrm{O}(27)$ & $81.0(6)$ \\
\hline $\mathrm{O}(11)-\mathrm{W}(1)-\mathrm{O}(26)$ & $82.8(6)$ & $\mathrm{O}(60)-\mathrm{W}(14)-\mathrm{O}(6)$ & $101.8(8)$ \\
\hline $\mathrm{O}(53)-\mathrm{W}(1)-\mathrm{O}(26)$ & $83.4(6)$ & $\mathrm{O}(60)-\mathrm{W}(14)-\mathrm{O}(48)$ & $98.6(8)$ \\
\hline $\mathrm{O}(15)-\mathrm{W}(2)-\mathrm{O}(23)$ & 102.1(8) & $\mathrm{O}(6)-\mathrm{W}(14)-\mathrm{O}(48)$ & $89.6(7)$ \\
\hline $\mathrm{O}(15)-\mathrm{W}(2)-\mathrm{O}(54)$ & $100.6(8)$ & $\mathrm{O}(60)-\mathrm{W}(14)-\mathrm{O}(51)$ & $101.2(8)$ \\
\hline $\mathrm{O}(23)-\mathrm{W}(2)-\mathrm{O}(54)$ & $88.9(7)$ & $\mathrm{O}(6)-\mathrm{W}(14)-\mathrm{O}(51)$ & $156.6(7)$ \\
\hline $\mathrm{O}(15)-\mathrm{W}(2)-\mathrm{O}(53)$ & $102.0(8)$ & $\mathrm{O}(48)-\mathrm{W}(14)-\mathrm{O}(51)$ & $91.2(7)$ \\
\hline $\mathrm{O}(23)-\mathrm{W}(2)-\mathrm{O}(53)$ & $83.3(7)$ & $\mathrm{O}(60)-\mathrm{W}(14)-\mathrm{O}(56)$ & $96.9(8)$ \\
\hline $\mathrm{O}(54)-\mathrm{W}(2)-\mathrm{O}(53)$ & $157.2(7)$ & $\mathrm{O}(6)-\mathrm{W}(14)-\mathrm{O}(56)$ & $84.7(7)$ \\
\hline $\mathrm{O}(15)-\mathrm{W}(2)-\mathrm{O}(50)$ & 103.4(9) & $\mathrm{O}(48)-\mathrm{W}(14)-\mathrm{O}(56)$ & $164.2(7)$ \\
\hline $\mathrm{O}(23)-\mathrm{W}(2)-\mathrm{O}(50)$ & $154.3(7)$ & $\mathrm{O}(51)-\mathrm{W}(14)-\mathrm{O}(56)$ & $88.3(7)$ \\
\hline $\mathrm{O}(54)-\mathrm{W}(2)-\mathrm{O}(50)$ & $88.3(7)$ & $\mathrm{O}(60)-\mathrm{W}(14)-\mathrm{O}(7)$ & $174.2(8)$ \\
\hline $\mathrm{O}(53)-\mathrm{W}(2)-\mathrm{O}(50)$ & $89.6(7)$ & $\mathrm{O}(6)-\mathrm{W}(14)-\mathrm{O}(7)$ & $83.5(6)$ \\
\hline $\mathrm{O}(15)-\mathrm{W}(2)-\mathrm{O}(17)$ & $173.2(8)$ & $\mathrm{O}(48)-\mathrm{W}(14)-\mathrm{O}(7)$ & $83.6(6)$ \\
\hline $\mathrm{O}(23)-\mathrm{W}(2)-\mathrm{O}(17)$ & $81.9(6)$ & $\mathrm{O}(51)-\mathrm{W}(14)-\mathrm{O}(7)$ & $73.3(6)$ \\
\hline $\mathrm{O}(54)-\mathrm{W}(2)-\mathrm{O}(17)$ & $73.8(6)$ & $\mathrm{O}(56)-\mathrm{W}(14)-\mathrm{O}(7)$ & $81.1(6)$ \\
\hline $\mathrm{O}(53)-\mathrm{W}(2)-\mathrm{O}(17)$ & $83.9(6)$ & $\mathrm{O}(21)-\mathrm{W}(15)-\mathrm{O}(20)$ & $101.7(8)$ \\
\hline
\end{tabular}




\begin{tabular}{|c|c|c|c|}
\hline $\mathrm{O}(50)-\mathrm{W}(2)-\mathrm{O}(17)$ & $72.9(6)$ & $\mathrm{O}(21)-\mathrm{W}(15)-\mathrm{O}(22)$ & 101.2(7) \\
\hline $\mathrm{O}(4)-\mathrm{W}(3)-\mathrm{O}(2)$ & $102.3(8)$ & $\mathrm{O}(20)-\mathrm{W}(15)-\mathrm{O}(22)$ & $157.1(7)$ \\
\hline $\mathrm{O}(4)-\mathrm{W}(3)-\mathrm{O}(6)$ & $101.0(8)$ & $\mathrm{O}(21)-\mathrm{W}(15)-\mathrm{O}(18)$ & $101.5(7)$ \\
\hline $\mathrm{O}(2)-\mathrm{W}(3)-\mathrm{O}(6)$ & $156.5(6)$ & $\mathrm{O}(20)-\mathrm{W}(15)-\mathrm{O}(18)$ & $90.9(7)$ \\
\hline $\mathrm{O}(4)-\mathrm{W}(3)-\mathrm{O}(59)$ & $96.7(7)$ & $\mathrm{O}(22)-\mathrm{W}(15)-\mathrm{O}(18)$ & $84.6(7)$ \\
\hline $\mathrm{O}(2)-\mathrm{W}(3)-\mathrm{O}(59)$ & $93.6(7)$ & $\mathrm{O}(21)-\mathrm{W}(15)-\mathrm{O}(54)$ & $99.8(7)$ \\
\hline $\mathrm{O}(6)-\mathrm{W}(3)-\mathrm{O}(59)$ & $86.3(7)$ & $\mathrm{O}(20)-\mathrm{W}(15)-\mathrm{O}(54)$ & $87.8(7)$ \\
\hline $\mathrm{O}(4)-\mathrm{W}(3)-\mathrm{O}(3)$ & 98.6(7) & $\mathrm{O}(22)-\mathrm{W}(15)-\mathrm{O}(54)$ & $88.2(7)$ \\
\hline $\mathrm{O}(2)-\mathrm{W}(3)-\mathrm{O}(3)$ & $86.9(6)$ & $\mathrm{O}(18)-\mathrm{W}(15)-\mathrm{O}(54)$ & $158.5(7)$ \\
\hline $\mathrm{O}(6)-\mathrm{W}(3)-\mathrm{O}(3)$ & $86.9(7)$ & $\mathrm{O}(21)-\mathrm{W}(15)-\mathrm{O}(17)$ & $172.2(7)$ \\
\hline $\mathrm{O}(59)-\mathrm{W}(3)-\mathrm{O}(3)$ & $164.2(7)$ & $\mathrm{O}(20)-\mathrm{W}(15)-\mathrm{O}(17)$ & $73.6(6)$ \\
\hline $\mathrm{O}(4)-\mathrm{W}(3)-\mathrm{O}(5)$ & $174.7(7)$ & $\mathrm{O}(22)-\mathrm{W}(15)-\mathrm{O}(17)$ & $83.6(6)$ \\
\hline $\mathrm{O}(2)-\mathrm{W}(3)-\mathrm{O}(5)$ & $72.5(6)$ & $\mathrm{O}(18)-\mathrm{W}(15)-\mathrm{O}(17)$ & $84.9(6)$ \\
\hline $\mathrm{O}(6)-\mathrm{W}(3)-\mathrm{O}(5)$ & $84.2(6)$ & $\mathrm{O}(54)-\mathrm{W}(15)-\mathrm{O}(17)$ & $74.1(6)$ \\
\hline $\mathrm{O}(59)-\mathrm{W}(3)-\mathrm{O}(5)$ & $83.1(6)$ & $\mathrm{O}(16)-\mathrm{W}(16)-\mathrm{O}(32)$ & $100.2(8)$ \\
\hline $\mathrm{O}(3)-\mathrm{W}(3)-\mathrm{O}(5)$ & $82.0(6)$ & $\mathrm{O}(16)-\mathrm{W}(16)-\mathrm{O}(33)$ & $100.9(8)$ \\
\hline $\mathrm{O}(52)-\mathrm{W}(4)-\mathrm{O}(51)$ & $100.2(8)$ & $\mathrm{O}(32)-\mathrm{W}(16)-\mathrm{O}(33)$ & $158.8(7)$ \\
\hline $\mathrm{O}(52)-\mathrm{W}(4)-\mathrm{O}(47)$ & $99.2(8)$ & $\mathrm{O}(16)-\mathrm{W}(16)-\mathrm{O}(47)$ & $99.0(8)$ \\
\hline $\mathrm{O}(51)-\mathrm{W}(4)-\mathrm{O}(47)$ & $90.5(7)$ & $\mathrm{O}(32)-\mathrm{W}(16)-\mathrm{O}(47)$ & $89.2(7)$ \\
\hline $\mathrm{O}(52)-\mathrm{W}(4)-\mathrm{O}(38)$ & $102.7(8)$ & $\mathrm{O}(33)-\mathrm{W}(16)-\mathrm{O}(47)$ & $89.5(7)$ \\
\hline $\mathrm{O}(51)-\mathrm{W}(4)-\mathrm{O}(38)$ & $156.9(7)$ & $\mathrm{O}(16)-\mathrm{W}(16)-\mathrm{O}(18)$ & $98.4(8)$ \\
\hline $\mathrm{O}(47)-\mathrm{W}(4)-\mathrm{O}(38)$ & $89.0(7)$ & $\mathrm{O}(32)-\mathrm{W}(16)-\mathrm{O}(18)$ & $91.3(7)$ \\
\hline $\mathrm{O}(52)-\mathrm{W}(4)-\mathrm{O}(43)$ & $96.0(8)$ & $\mathrm{O}(33)-\mathrm{W}(16)-\mathrm{O}(18)$ & $83.8(7)$ \\
\hline $\mathrm{O}(51)-\mathrm{W}(4)-\mathrm{O}(43)$ & $88.8(7)$ & $\mathrm{O}(47)-\mathrm{W}(16)-\mathrm{O}(18)$ & $162.3(7)$ \\
\hline $\mathrm{O}(47)-\mathrm{W}(4)-\mathrm{O}(43)$ & $164.7(7)$ & $\mathrm{O}(16)-\mathrm{W}(16)-\mathrm{O}(27)$ & $173.7(7)$ \\
\hline $\mathrm{O}(38)-\mathrm{W}(4)-\mathrm{O}(43)$ & $85.8(7)$ & $\mathrm{O}(32)-\mathrm{W}(16)-\mathrm{O}(27)$ & $73.8(6)$ \\
\hline $\mathrm{O}(52)-\mathrm{W}(4)-\mathrm{O}(7)$ & $173.6(8)$ & $\mathrm{O}(33)-\mathrm{W}(16)-\mathrm{O}(27)$ & $85.1(6)$ \\
\hline $\mathrm{O}(51)-\mathrm{W}(4)-\mathrm{O}(7)$ & $73.8(6)$ & $\mathrm{O}(47)-\mathrm{W}(16)-\mathrm{O}(27)$ & $82.8(6)$ \\
\hline $\mathrm{O}(47)-\mathrm{W}(4)-\mathrm{O}(7)$ & $83.2(6)$ & $\mathrm{O}(18)-\mathrm{W}(16)-\mathrm{O}(27)$ & $80.3(6)$ \\
\hline $\mathrm{O}(38)-\mathrm{W}(4)-\mathrm{O}(7)$ & $83.2(6)$ & $\mathrm{O}(49)-\mathrm{W}(17)-\mathrm{O}(44)$ & $103.2(8)$ \\
\hline $\mathrm{O}(43)-\mathrm{W}(4)-\mathrm{O}(7)$ & $82.0(6)$ & $\mathrm{O}(49)-\mathrm{W}(17)-\mathrm{O}(45)$ & $99.2(8)$ \\
\hline $\mathrm{O}(42)-\mathrm{W}(5)-\mathrm{O}(40)$ & $102.2(8)$ & $\mathrm{O}(44)-\mathrm{W}(17)-\mathrm{O}(45)$ & $88.4(7)$ \\
\hline $\mathrm{O}(42)-\mathrm{W}(5)-\mathrm{O}(43)$ & $103.0(8)$ & $\mathrm{O}(49)-\mathrm{W}(17)-\mathrm{O}(39)$ & $102.4(8)$ \\
\hline $\mathrm{O}(40)-\mathrm{W}(5)-\mathrm{O}(43)$ & $87.0(7)$ & $\mathrm{O}(44)-\mathrm{W}(17)-\mathrm{O}(39)$ & $86.4(7)$ \\
\hline $\mathrm{O}(42)-\mathrm{W}(5)-\mathrm{O}(9)$ & $101.3(8)$ & $\mathrm{O}(45)-\mathrm{W}(17)-\mathrm{O}(39)$ & $158.4(7)$ \\
\hline $\mathrm{O}(40)-\mathrm{W}(5)-\mathrm{O}(9)$ & $156.4(7)$ & $\mathrm{O}(49)-\mathrm{W}(17)-\mathrm{O}(41)$ & $98.3(8)$ \\
\hline $\mathrm{O}(43)-\mathrm{W}(5)-\mathrm{O}(9)$ & $86.9(7)$ & $\mathrm{O}(44)-\mathrm{W}(17)-\mathrm{O}(41)$ & $158.5(8)$ \\
\hline $\mathrm{O}(42)-\mathrm{W}(5)-\mathrm{O}(41)$ & $100.1(8)$ & $\mathrm{O}(45)-\mathrm{W}(17)-\mathrm{O}(41)$ & $88.1(7)$ \\
\hline $\mathrm{O}(40)-\mathrm{W}(5)-\mathrm{O}(41)$ & $90.0(7)$ & $\mathrm{O}(39)-\mathrm{W}(17)-\mathrm{O}(41)$ & $89.0(8)$ \\
\hline $\mathrm{O}(43)-\mathrm{W}(5)-\mathrm{O}(41)$ & $156.9(8)$ & $\mathrm{O}(49)-\mathrm{W}(17)-\mathrm{O}(8)$ & $169.1(7)$ \\
\hline $\mathrm{O}(9)-\mathrm{W}(5)-\mathrm{O}(41)$ & $86.8(8)$ & $\mathrm{O}(44)-\mathrm{W}(17)-\mathrm{O}(8)$ & $85.0(7)$ \\
\hline $\mathrm{O}(42)-\mathrm{W}(5)-\mathrm{O}(8)$ & $170.5(7)$ & $\mathrm{O}(45)-\mathrm{W}(17)-\mathrm{O}(8)$ & $73.6(6)$ \\
\hline $\mathrm{O}(40)-\mathrm{W}(5)-\mathrm{O}(8)$ & $84.0(6)$ & $\mathrm{O}(39)-\mathrm{W}(17)-\mathrm{O}(8)$ & $85.0(6)$ \\
\hline
\end{tabular}




\begin{tabular}{|c|c|c|c|}
\hline $\mathrm{O}(43)-\mathrm{W}(5)-\mathrm{O}(8)$ & $84.3(7)$ & $\mathrm{O}(41)-\mathrm{W}(17)-\mathrm{O}(8)$ & 73.7(7) \\
\hline $\mathrm{O}(9)-\mathrm{W}(5)-\mathrm{O}(8)$ & $72.8(6)$ & $\mathrm{O}(30)-\mathrm{W}(18)-\mathrm{O}(3)$ & $99.8(8)$ \\
\hline $\mathrm{O}(41)-\mathrm{W}(5)-\mathrm{O}(8)$ & $72.6(7)$ & $\mathrm{O}(30)-\mathrm{W}(18)-\mathrm{O}(29)$ & $101.0(8)$ \\
\hline $\mathrm{O}(57)-\mathrm{W}(6)-\mathrm{O}(11)$ & $102.5(8)$ & $\mathrm{O}(3)-\mathrm{W}(18)-\mathrm{O}(29)$ & $91.4(7)$ \\
\hline $\mathrm{O}(57)-\mathrm{W}(6)-\mathrm{O}(13)$ & $96.6(8)$ & $\mathrm{O}(30)-\mathrm{W}(18)-\mathrm{O}(28)$ & $102.6(7)$ \\
\hline $\mathrm{O}(11)-\mathrm{W}(6)-\mathrm{O}(13)$ & $87.3(7)$ & $\mathrm{O}(3)-\mathrm{W}(18)-\mathrm{O}(28)$ & $91.0(7)$ \\
\hline $\mathrm{O}(57)-\mathrm{W}(6)-\mathrm{O}(23)$ & $99.6(8)$ & $\mathrm{O}(29)-\mathrm{W}(18)-\mathrm{O}(28)$ & $155.5(7)$ \\
\hline $\mathrm{O}(11)-\mathrm{W}(6)-\mathrm{O}(23)$ & $85.2(7)$ & $\mathrm{O}(30)-\mathrm{W}(18)-\mathrm{O}(19)$ & $96.4(8)$ \\
\hline $\mathrm{O}(13)-\mathrm{W}(6)-\mathrm{O}(23)$ & 163.3(7) & $\mathrm{O}(3)-\mathrm{W}(18)-\mathrm{O}(19)$ & $163.8(6)$ \\
\hline $\mathrm{O}(57)-\mathrm{W}(6)-\mathrm{O}(58)$ & $99.9(8)$ & $\mathrm{O}(29)-\mathrm{W}(18)-\mathrm{O}(19)$ & $84.9(7)$ \\
\hline $\mathrm{O}(11)-\mathrm{W}(6)-\mathrm{O}(58)$ & $157.6(7)$ & $\mathrm{O}(28)-\mathrm{W}(18)-\mathrm{O}(19)$ & $86.1(6)$ \\
\hline $\mathrm{O}(13)-\mathrm{W}(6)-\mathrm{O}(58)$ & $89.5(7)$ & $\mathrm{O}(30)-\mathrm{W}(18)-\mathrm{O}(26)$ & $173.3(7)$ \\
\hline $\mathrm{O}(23)-\mathrm{W}(6)-\mathrm{O}(58)$ & $91.7(7)$ & $\mathrm{O}(3)-\mathrm{W}(18)-\mathrm{O}(26)$ & $83.4(6)$ \\
\hline $\mathrm{O}(57)-\mathrm{W}(6)-\mathrm{O}(25)$ & 173.1(7) & $\mathrm{O}(29)-\mathrm{W}(18)-\mathrm{O}(26)$ & $84.7(7)$ \\
\hline $\mathrm{O}(11)-\mathrm{W}(6)-\mathrm{O}(25)$ & $84.4(6)$ & $\mathrm{O}(28)-\mathrm{W}(18)-\mathrm{O}(26)$ & $71.4(6)$ \\
\hline $\mathrm{O}(13)-\mathrm{W}(6)-\mathrm{O}(25)$ & $82.6(6)$ & $\mathrm{O}(19)-\mathrm{W}(18)-\mathrm{O}(26)$ & $80.5(6)$ \\
\hline $\mathrm{O}(23)-\mathrm{W}(6)-\mathrm{O}(25)$ & $81.9(6)$ & $\mathrm{O}(13)-\mathrm{W}(8)-\mathrm{O}(39)$ & $163.3(7)$ \\
\hline $\mathrm{O}(58)-\mathrm{W}(6)-\mathrm{O}(25)$ & $73.2(6)$ & $\mathrm{O}(34)-\mathrm{W}(8)-\mathrm{O}(39)$ & $84.9(7)$ \\
\hline $\mathrm{O}(46)-\mathrm{W}(7)-\mathrm{O}(56)$ & 103.1(8) & $\mathrm{O}(62)-\mathrm{W}(8)-\mathrm{O}(36)$ & $174.9(6)$ \\
\hline $\mathrm{O}(46)-\mathrm{W}(7)-\mathrm{O}(9)$ & 102.1(8) & $\mathrm{O}(35)-\mathrm{W}(8)-\mathrm{O}(36)$ & $73.9(6)$ \\
\hline $\mathrm{O}(56)-\mathrm{W}(7)-\mathrm{O}(9)$ & $89.7(7)$ & $\mathrm{O}(13)-\mathrm{W}(8)-\mathrm{O}(36)$ & $83.2(6)$ \\
\hline $\mathrm{O}(46)-\mathrm{W}(7)-\mathrm{O}(59)$ & $101.8(8)$ & $\mathrm{O}(34)-\mathrm{W}(8)-\mathrm{O}(36)$ & $83.6(6)$ \\
\hline $\mathrm{O}(56)-\mathrm{W}(7)-\mathrm{O}(59)$ & $85.5(7)$ & $\mathrm{O}(39)-\mathrm{W}(8)-\mathrm{O}(36)$ & $81.1(6)$ \\
\hline $\mathrm{O}(9)-\mathrm{W}(7)-\mathrm{O}(59)$ & 156.1(7) & $\mathrm{O}(37)-\mathrm{W}(9)-\mathrm{O}(33)$ & $101.0(7)$ \\
\hline $\mathrm{O}(46)-\mathrm{W}(7)-\mathrm{O}(45)$ & 101.7(8) & $\mathrm{O}(37)-\mathrm{W}(9)-\mathrm{O}(58)$ & $99.7(7)$ \\
\hline $\mathrm{O}(56)-\mathrm{W}(7)-\mathrm{O}(45)$ & 154.8(7) & $\mathrm{O}(33)-\mathrm{W}(9)-\mathrm{O}(58)$ & $159.3(7)$ \\
\hline $\mathrm{O}(9)-\mathrm{W}(7)-\mathrm{O}(45)$ & $89.4(7)$ & $\mathrm{O}(37)-\mathrm{W}(9)-\mathrm{O}(22)$ & $100.4(7)$ \\
\hline $\mathrm{O}(59)-\mathrm{W}(7)-\mathrm{O}(45)$ & $85.2(7)$ & $\mathrm{O}(33)-\mathrm{W}(9)-\mathrm{O}(22)$ & $86.0(7)$ \\
\hline $\mathrm{O}(46)-\mathrm{W}(7)-\mathrm{O}(8)$ & 171.3(8) & $\mathrm{O}(58)-\mathrm{W}(9)-\mathrm{O}(22)$ & $89.9(7)$ \\
\hline $\mathrm{O}(56)-\mathrm{W}(7)-\mathrm{O}(8)$ & $84.1(6)$ & $\mathrm{O}(37)-\mathrm{W}(9)-\mathrm{O}(14)$ & $97.7(8)$ \\
\hline $\mathrm{O}(9)-\mathrm{W}(7)-\mathrm{O}(8)$ & $72.7(7)$ & $\mathrm{O}(33)-\mathrm{W}(9)-\mathrm{O}(14)$ & $87.7(8)$ \\
\hline $\mathrm{O}(59)-\mathrm{W}(7)-\mathrm{O}(8)$ & $83.5(7)$ & $\mathrm{O}(58)-\mathrm{W}(9)-\mathrm{O}(14)$ & $89.9(8)$ \\
\hline $\mathrm{O}(45)-\mathrm{W}(7)-\mathrm{O}(8)$ & $71.6(6)$ & $\mathrm{O}(22)-\mathrm{W}(9)-\mathrm{O}(14)$ & $161.7(7)$ \\
\hline $\mathrm{O}(62)-\mathrm{W}(8)-\mathrm{O}(35)$ & 101.2(7) & $\mathrm{O}(37)-\mathrm{W}(9)-\mathrm{O}(25)$ & $172.9(7)$ \\
\hline $\mathrm{O}(62)-\mathrm{W}(8)-\mathrm{O}(13)$ & $98.6(7)$ & $\mathrm{O}(33)-\mathrm{W}(9)-\mathrm{O}(25)$ & $86.0(6)$ \\
\hline $\mathrm{O}(35)-\mathrm{W}(8)-\mathrm{O}(13)$ & $90.5(7)$ & $\mathrm{O}(58)-\mathrm{W}(9)-\mathrm{O}(25)$ & $73.3(6)$ \\
\hline $\mathrm{O}(62)-\mathrm{W}(8)-\mathrm{O}(34)$ & 101.2(7) & $\mathrm{O}(22)-\mathrm{W}(9)-\mathrm{O}(25)$ & $80.5(6)$ \\
\hline $\mathrm{O}(35)-\mathrm{W}(8)-\mathrm{O}(34)$ & $157.6(7)$ & $\mathrm{O}(14)-\mathrm{W}(9)-\mathrm{O}(25)$ & $82.0(7)$ \\
\hline $\mathrm{O}(13)-\mathrm{W}(8)-\mathrm{O}(34)$ & $87.7(6)$ & $\mathrm{O}(12)-\mathrm{W}(10)-\mathrm{O}(19)$ & $102.2(8)$ \\
\hline $\mathrm{O}(62)-\mathrm{W}(8)-\mathrm{O}(39)$ & $97.5(7)$ & $\mathrm{O}(12)-\mathrm{W}(10)-\mathrm{O}(24)$ & $102.6(8)$ \\
\hline $\mathrm{O}(35)-\mathrm{W}(8)-\mathrm{O}(39)$ & $90.6(7)$ & $\mathrm{O}(19)-\mathrm{W}(10)-\mathrm{O}(24)$ & $87.4(7)$ \\
\hline $\mathrm{O}(24)-\mathrm{W}(10)-\mathrm{O}(20)$ & $87.4(7)$ & $\mathrm{O}(12)-\mathrm{W}(10)-\mathrm{O}(50)$ & $101.6(8)$ \\
\hline $\mathrm{O}(50)-\mathrm{W}(10)-\mathrm{O}(20)$ & $85.3(7)$ & $\mathrm{O}(19)-\mathrm{W}(10)-\mathrm{O}(50)$ & $89.5(7)$ \\
\hline
\end{tabular}




\begin{tabular}{|l|l|l|l|}
\hline $\mathrm{O}(12)-\mathrm{W}(10)-\mathrm{O}(17)$ & $171.7(7)$ & $\mathrm{O}(24)-\mathrm{W}(10)-\mathrm{O}(50)$ & $155.8(7)$ \\
\hline $\mathrm{O}(19)-\mathrm{W}(10)-\mathrm{O}(17)$ & $84.0(6)$ & $\mathrm{O}(12)-\mathrm{W}(10)-\mathrm{O}(20)$ & $102.9(8)$ \\
\hline $\mathrm{O}(24)-\mathrm{W}(10)-\mathrm{O}(17)$ & $83.2(6)$ & $\mathrm{O}(19)-\mathrm{W}(10)-\mathrm{O}(20)$ & $154.9(7)$ \\
\hline $\mathrm{O}(50)-\mathrm{W}(10)-\mathrm{O}(17)$ & $72.6(6)$ & $\mathrm{O}(61)-\mathrm{W}(11)-\mathrm{O}(14)$ & $98.6(8)$ \\
\hline $\mathrm{O}(20)-\mathrm{W}(10)-\mathrm{O}(17)$ & $71.0(6)$ & $\mathrm{O}(61)-\mathrm{W}(11)-\mathrm{O}(38)$ & $103.2(8)$ \\
\hline $\mathrm{O}(14)-\mathrm{W}(11)-\mathrm{O}(40)$ & $164.7(7)$ & $\mathrm{O}(14)-\mathrm{W}(11)-\mathrm{O}(38)$ & $89.4(8)$ \\
\hline
\end{tabular}

Table S4. Selected Bond Lengths $(\AA)$ and Bond Angles $\left({ }^{\circ}\right)$ for Compound 3.

\begin{tabular}{|l|l|l|l|}
\hline $\mathrm{N}(16)-\operatorname{Ag}(7)$ & $2.172(14)$ & $\mathrm{O}(41)-\mathrm{P}(2)$ & $1.545(9)$ \\
\hline $\mathrm{N}(1)-\operatorname{Ag}(6)$ & $2.125(12)$ & $\mathrm{O}(41)-\mathrm{W}(8)$ & $2.344(9)$ \\
\hline $\mathrm{N}(2)-\operatorname{Ag}(1)$ & $2.388(12)$ & $\mathrm{O}(41)-\mathrm{W}(7)$ & $2.372(9)$ \\
\hline $\mathrm{N}(3)-\operatorname{Ag}(1)$ & $2.347(15)$ & $\mathrm{O}(42)-\mathrm{P}(2)$ & $1.521(9)$ \\
\hline $\mathrm{N}(4)-\operatorname{Ag}(7)$ & $2.264(14)$ & $\mathrm{O}(42)-\mathrm{W}(5)$ & $2.347(9)$ \\
\hline $\mathrm{N}(5)-\operatorname{Ag}(7)$ & $2.441(12)$ & $\mathrm{O}(42)-\mathrm{W}(6)$ & $2.380(8)$ \\
\hline $\mathrm{N}(6)-\operatorname{Ag}(3)$ & $2.141(13)$ & $\mathrm{O}(43)-\mathrm{W}(2)$ & $1.930(9)$ \\
\hline $\mathrm{N}(7)-\operatorname{Ag}(3)$ & $2.190(13)$ & $\mathrm{O}(43)-\mathrm{W}(3)$ & $1.930(9)$ \\
\hline $\mathrm{N}(8)-\operatorname{Ag}(2)$ & $2.178(12)$ & $\mathrm{O}(44)-\mathrm{W}(12)$ & $1.902(11)$ \\
\hline $\mathrm{N}(9)-\operatorname{Ag}(1)$ & $2.181(12)$ & $\mathrm{O}(44)-\mathrm{W}(17)$ & $1.905(11)$ \\
\hline $\mathrm{N}(10)-\operatorname{Ag}(4)$ & $2.102(14)$ & $\mathrm{O}(45)-\mathrm{W}(14)$ & $1.887(8)$ \\
\hline $\mathrm{N}(11)-\operatorname{Ag}(4)$ & $2.093(11)$ & $\mathrm{O}(45)-\mathrm{W}(13)$ & $1.920(8)$ \\
\hline $\mathrm{N}(12)-\operatorname{Ag}(6)$ & $2.106(11)$ & $\mathrm{O}(46)-\mathrm{P}(2)$ & $1.595(9)$ \\
\hline $\mathrm{N}(13)-\operatorname{Ag}(5)$ & $2.219(13)$ & $\mathrm{O}(46)-\mathrm{W}(1)$ & $2.356(9)$ \\
\hline $\mathrm{N}(17)-\operatorname{Ag}(2)+1$ & $2.192(13)$ & $\mathrm{O}(46)-\mathrm{W}(3)$ & $2.374(8)$ \\
\hline $\mathrm{N}(20)-\operatorname{Ag}(5)$ & $2.262(13)$ & $\mathrm{O}(46)-\mathrm{W}(2)$ & $2.381(8)$ \\
\hline $\mathrm{O}(1)-\mathrm{W}(12)$ & $1.699(9)$ & $\mathrm{O}(47)-\mathrm{W}(5)$ & $1.898(9)$ \\
\hline $\mathrm{O}(2)-\mathrm{W}(10)$ & $1.727(13)$ & $\mathrm{O}(47)-\mathrm{W}(6)$ & $1.899(10)$ \\
\hline $\mathrm{O}(3)-\mathrm{W}(11)$ & $1.704(9)$ & $\mathrm{O}(48)-\mathrm{W}(2)$ & $1.899(9)$ \\
\hline $\mathrm{O}(4)-\mathrm{W}(15)$ & $1.690(9)$ & $\mathrm{O}(48)-\mathrm{W}(7)$ & $1.923(9)$ \\
\hline $\mathrm{O}(5)-\mathrm{W}(16)$ & $1.696(9)$ & $\mathrm{O}(49)-\mathrm{W}(4)$ & $1.900(10)$ \\
\hline $\mathrm{O}(6)-\mathrm{W}(18)$ & $1.722(9)$ & $\mathrm{O}(49)-\mathrm{W}(9)$ & $1.914(10)$ \\
\hline $\mathrm{O}(7)-\mathrm{W}(14)$ & $1.719(10)$ & $\mathrm{O}(50)-\mathrm{P}(1)$ & $1.529(10)$ \\
\hline $\mathrm{O}(8)-\mathrm{W}(13)$ & $1.718(11)$ & $\mathrm{O}(50)-\mathrm{W}(17)$ & $2.335(9)$ \\
\hline $\mathrm{O}(9)-\mathrm{W}(7)$ & $1.723(9)$ & $\mathrm{O}(50)-\mathrm{W}(18)$ & $2.384(8)$ \\
\hline $\mathrm{O}(10)-\mathrm{W}(6)$ & $1.698(8)$ & $\mathrm{O}(51)-\mathrm{P}(2)$ & $1.525(9)$ \\
\hline $\mathrm{O}(11)-\mathrm{W}(8)$ & $1.701(11)$ & $\mathrm{O}(51)-\mathrm{W}(4)$ & $2.354(9)$ \\
\hline $\mathrm{O}(12)-\mathrm{W}(4)$ & $1.729(10)$ & $\mathrm{O}(51)-\mathrm{W}(9)$ & $2.367(9)$ \\
\hline $\mathrm{O}(13)-\mathrm{W}(5)$ & $1.704(10)$ & $\mathrm{O}(52)-\mathrm{W}(4)$ & $1.905(9)$ \\
\hline $\mathrm{O}(14)-\mathrm{W}(9)$ & $1.706(9)$ & $\mathrm{O}(52)-\mathrm{W}(3)$ & $1.916(9)$ \\
\hline $\mathrm{O}(15)-\mathrm{P}(1)$ & $1.502(9)$ & $\mathrm{O}(53)-\mathrm{W}(18)$ & $1.878(9)$ \\
\hline $\mathrm{O}(15)-\mathrm{W}(13)$ & $2.349(10)$ & $\mathrm{O}(53)-\mathrm{W}(7)$ & $1.922(9)$ \\
\hline $\mathrm{O}(15)-\mathrm{W}(15)$ & $2.413(8)$ & $\mathrm{O}(54)-\mathrm{W}(14)$ & $1.906(9)$ \\
\hline $\mathrm{O}(16)-\mathrm{W}(1)$ & $1.744(11)$ & $\mathrm{O}(54)-\mathrm{W}(16)$ & $1.915(10)$ \\
\hline $\mathrm{O}(17)-\mathrm{W}(3)$ & $1.711(9)$ & $\mathrm{O}(55)-\mathrm{W}(18)$ & $1.884(10)$ \\
\hline & & & \\
\hline
\end{tabular}




\begin{tabular}{|c|c|c|c|}
\hline $\mathrm{O}(18)-\mathrm{W}(8)$ & $1.884(9)$ & $\mathrm{O}(55)-\mathrm{W}(11)$ & $1.932(10)$ \\
\hline $\mathrm{O}(18)-\mathrm{W}(17)$ & $1.906(10)$ & $\mathrm{O}(56)-\mathrm{W}(14)$ & $1.873(9)$ \\
\hline $\mathrm{O}(19)-\mathrm{W}(9)$ & $1.884(9)$ & $\mathrm{O}(56)-\mathrm{W}(4)$ & $1.926(9)$ \\
\hline $\mathrm{O}(19)-\mathrm{W}(16)$ & $1.904(9)$ & $\mathrm{O}(57)-\mathrm{W}(7)$ & $1.902(9)$ \\
\hline $\mathrm{O}(20)-\mathrm{W}(1)$ & $1.878(9)$ & $\mathrm{O}(57)-\mathrm{W}(8)$ & $1.909(8)$ \\
\hline $\mathrm{O}(20)-\mathrm{W}(8)$ & $1.930(9)$ & $\mathrm{O}(58)-\mathrm{W}(4)$ & $1.895(8)$ \\
\hline $\mathrm{O}(21)-\mathrm{W}(1)$ & $1.878(9)$ & $\mathrm{O}(58)-\mathrm{W}(5)$ & $1.909(8)$ \\
\hline $\mathrm{O}(21)-\mathrm{W}(9)$ & $1.952(9)$ & $\mathrm{O}(59)-\mathrm{W}(2)$ & $1.726(10)$ \\
\hline $\mathrm{O}(22)-\mathrm{W}(6)$ & $1.910(10)$ & $\mathrm{O}(59)-\operatorname{Ag}(5)$ & $2.571(11)$ \\
\hline $\mathrm{O}(22)-\mathrm{W}(2)$ & $1.913(10)$ & $\mathrm{O}(60)-\mathrm{W}(18)$ & $1.897(8)$ \\
\hline $\mathrm{O}(23)-\mathrm{W}(5)$ & $1.902(10)$ & $\mathrm{O}(60)-\mathrm{W}(17)$ & $1.943(8)$ \\
\hline $\mathrm{O}(23)-\mathrm{W}(3)$ & $1.923(10)$ & $\mathrm{O}(61)-\mathrm{W}(8)$ & $1.882(9)$ \\
\hline $\mathrm{O}(24)-\mathrm{W}(3)$ & $1.899(11)$ & $\mathrm{O}(61)-\mathrm{W}(9)$ & $1.917(10)$ \\
\hline $\mathrm{O}(24)-\mathrm{W}(1)$ & $1.956(9)$ & $\mathrm{O}(62)-\mathrm{W}(17)$ & $1.723(9)$ \\
\hline $\mathrm{O}(25)-\mathrm{W}(2)$ & $1.890(9)$ & $\operatorname{Ag}(1)-\operatorname{Ag}(2)$ & $3.336(2)$ \\
\hline $\mathrm{O}(25)-\mathrm{W}(1)$ & $1.936(9)$ & $\operatorname{Ag}(2)-N(17) \# 2$ & $2.192(13)$ \\
\hline $\mathrm{O}(26)-\mathrm{W}(7)$ & $1.894(10)$ & $\operatorname{Ag}(3)-\operatorname{Ag}(6)$ & $3.350(2)$ \\
\hline $\mathrm{O}(26)-\mathrm{W}(6)$ & $1.895(9)$ & $\operatorname{Ag}(4)-\operatorname{Ag}(5)$ & $3.153(2)$ \\
\hline $\mathrm{O}(27)-\mathrm{W}(13)$ & $1.902(9)$ & $\mathrm{O}(33)-\mathrm{W}(12)$ & $1.916(9)$ \\
\hline $\mathrm{O}(27)-\mathrm{W}(5)$ & $1.926(9)$ & $\mathrm{O}(33)-\mathrm{W}(16)$ & $1.916(9)$ \\
\hline $\mathrm{O}(28)-\mathrm{W}(15)$ & $1.898(9)$ & $\mathrm{O}(34)-\mathrm{W}(17)$ & $1.871(9)$ \\
\hline $\mathrm{O}(28)-\mathrm{W}(6)$ & $1.901(9)$ & $\mathrm{O}(34)-\mathrm{W}(16)$ & $1.908(10)$ \\
\hline $\mathrm{O}(29)-\mathrm{W}(11)$ & $1.872(9)$ & $\mathrm{O}(35)-\mathrm{P}(1)$ & $1.575(9)$ \\
\hline $\mathrm{O}(29)-\mathrm{W}(15)$ & $1.947(9)$ & $\mathrm{O}(35)-\mathrm{W}(11)$ & $2.371(8)$ \\
\hline $\mathrm{O}(30)-\mathrm{W}(10)$ & $1.893(10)$ & $\mathrm{O}(35)-\mathrm{W}(10)$ & $2.387(10)$ \\
\hline $\mathrm{O}(30)-\mathrm{W}(13)$ & $1.940(10)$ & $\mathrm{O}(35)-\mathrm{W}(12)$ & $2.404(8)$ \\
\hline $\mathrm{O}(31)-\mathrm{W}(12)$ & $1.917(9)$ & $\mathrm{O}(36)-\mathrm{W}(10)$ & $1.888(9)$ \\
\hline $\mathrm{O}(31)-\mathrm{W}(11)$ & $1.928(9)$ & $\mathrm{O}(36)-\mathrm{W}(14)$ & $1.951(9)$ \\
\hline $\mathrm{O}(32)-\mathrm{W}(10)$ & $1.908(9)$ & $\mathrm{O}(37)-\mathrm{W}(11)$ & $1.911(12)$ \\
\hline $\mathrm{O}(32)-\mathrm{W}(12)$ & $1.933(11)$ & $\mathrm{O}(37)-\mathrm{W}(10)$ & $1.951(10)$ \\
\hline $\mathrm{O}(39)-\mathrm{W}(13)$ & $1.904(9)$ & $\mathrm{O}(38)-\mathrm{P}(1)$ & $1.532(8)$ \\
\hline $\mathrm{O}(39)-\mathrm{W}(15)$ & $1.927(10)$ & $\mathrm{O}(38)-\mathrm{W}(14)$ & $2.354(9)$ \\
\hline $\mathrm{O}(40)-\mathrm{W}(18)$ & $1.898(10)$ & $\mathrm{O}(38)-\mathrm{W}(16)$ & $2.401(9)$ \\
\hline $\mathrm{O}(15)-\mathrm{P}(1)-\mathrm{O}(50)$ & $112.1(5)$ & $\mathrm{O}(45)-\mathrm{W}(13)-\mathrm{O}(15)$ & $84.3(4)$ \\
\hline $\mathrm{O}(15)-\mathrm{P}(1)-\mathrm{O}(38)$ & $111.5(5)$ & $\mathrm{O}(30)-\mathrm{W}(13)-\mathrm{O}(15)$ & $82.0(4)$ \\
\hline $\mathrm{O}(50)-\mathrm{P}(1)-\mathrm{O}(38)$ & $111.8(6)$ & $\mathrm{O}(7)-\mathrm{W}(14)-\mathrm{O}(56)$ & $98.4(5)$ \\
\hline $\mathrm{O}(15)-\mathrm{P}(1)-\mathrm{O}(35)$ & $107.5(5)$ & $\mathrm{O}(7)-\mathrm{W}(14)-\mathrm{O}(45)$ & $100.3(5)$ \\
\hline $\mathrm{O}(50)-\mathrm{P}(1)-\mathrm{O}(35)$ & $106.1(5)$ & $\mathrm{O}(56)-\mathrm{W}(14)-\mathrm{O}(45)$ & $90.7(4)$ \\
\hline $\mathrm{O}(38)-\mathrm{P}(1)-\mathrm{O}(35)$ & $107.5(5)$ & $\mathrm{O}(7)-\mathrm{W}(14)-\mathrm{O}(54)$ & $100.7(4)$ \\
\hline $\mathrm{O}(42)-\mathrm{P}(2)-\mathrm{O}(51)$ & $112.4(6)$ & $\mathrm{O}(56)-\mathrm{W}(14)-\mathrm{O}(54)$ & $90.1(4)$ \\
\hline $\mathrm{O}(42)-\mathrm{P}(2)-\mathrm{O}(41)$ & $112.5(5)$ & $\mathrm{O}(45)-\mathrm{W}(14)-\mathrm{O}(54)$ & $158.6(4)$ \\
\hline $\mathrm{O}(51)-\mathrm{P}(2)-\mathrm{O}(41)$ & $112.6(5)$ & $\mathrm{O}(7)-\mathrm{W}(14)-\mathrm{O}(36)$ & $96.5(5)$ \\
\hline
\end{tabular}




\begin{tabular}{|c|c|c|c|}
\hline $\mathrm{O}(42)-\mathrm{P}(2)-\mathrm{O}(46)$ & $107.2(5)$ & $\mathrm{O}(56)-\mathrm{W}(14)-\mathrm{O}(36)$ & $164.9(4)$ \\
\hline $\mathrm{O}(51)-\mathrm{P}(2)-\mathrm{O}(46)$ & $105.7(5)$ & $\mathrm{O}(45)-\mathrm{W}(14)-\mathrm{O}(36)$ & $84.7(4)$ \\
\hline $\mathrm{O}(41)-\mathrm{P}(2)-\mathrm{O}(46)$ & $105.9(6)$ & $\mathrm{O}(54)-\mathrm{W}(14)-\mathrm{O}(36)$ & $89.0(4)$ \\
\hline $\mathrm{N}(9)-\operatorname{Ag}(1)-\mathrm{N}(3)$ & $145.0(5)$ & $\mathrm{O}(7)-\mathrm{W}(14)-\mathrm{O}(38)$ & $174.8(4)$ \\
\hline $\mathrm{N}(9)-\mathrm{Ag}(1)-\mathrm{N}(2)$ & $134.1(4)$ & $\mathrm{O}(56)-\mathrm{W}(14)-\mathrm{O}(38)$ & $83.2(4)$ \\
\hline $\mathrm{N}(3)-\mathrm{Ag}(1)-\mathrm{N}(2)$ & $71.3(5)$ & $\mathrm{O}(45)-\mathrm{W}(14)-\mathrm{O}(38)$ & $84.5(4)$ \\
\hline $\mathrm{N}(8)-\mathrm{Ag}(2)-\mathrm{N}(17) \# 2$ & $162.4(5)$ & $\mathrm{O}(54)-\mathrm{W}(14)-\mathrm{O}(38)$ & $74.3(3)$ \\
\hline $\mathrm{N}(6)-\mathrm{Ag}(3)-\mathrm{N}(7)$ & $157.9(5)$ & $\mathrm{O}(36)-\mathrm{W}(14)-\mathrm{O}(38)$ & $82.1(4)$ \\
\hline $\mathrm{N}(11)-\mathrm{Ag}(4)-\mathrm{N}(10)$ & $170.6(5)$ & $\mathrm{O}(4)-\mathrm{W}(15)-\mathrm{O}(28)$ & $100.6(4)$ \\
\hline $\mathrm{N}(13)-\operatorname{Ag}(5)-\mathrm{N}(20)$ & $163.9(5)$ & $\mathrm{O}(4)-\mathrm{W}(15)-\mathrm{O}(40)$ & $103.2(5)$ \\
\hline $\mathrm{N}(13)-\mathrm{Ag}(5)-\mathrm{O}(59)$ & $104.2(4)$ & $\mathrm{O}(28)-\mathrm{W}(15)-\mathrm{O}(40)$ & $89.8(4)$ \\
\hline $\mathrm{N}(20)-\mathrm{Ag}(5)-\mathrm{O}(59)$ & 91.1(4) & $\mathrm{O}(4)-\mathrm{W}(15)-\mathrm{O}(39)$ & $99.3(5)$ \\
\hline $\mathrm{N}(12)-\mathrm{Ag}(6)-\mathrm{N}(1)$ & $173.7(5)$ & $\mathrm{O}(28)-\mathrm{W}(15)-\mathrm{O}(39)$ & $89.4(4)$ \\
\hline $\mathrm{N}(16)-\operatorname{Ag}(7)-\mathrm{N}(4)$ & $167.9(5)$ & $\mathrm{O}(40)-\mathrm{W}(15)-\mathrm{O}(39)$ & $157.2(4)$ \\
\hline $\mathrm{N}(16)-\operatorname{Ag}(7)-\mathrm{N}(5)$ & $113.3(5)$ & $\mathrm{O}(4)-\mathrm{W}(15)-\mathrm{O}(29)$ & $97.1(4)$ \\
\hline $\mathrm{N}(4)-\operatorname{Ag}(7)-\mathrm{N}(5)$ & $74.0(5)$ & $\mathrm{O}(28)-\mathrm{W}(15)-\mathrm{O}(29)$ & $162.3(4)$ \\
\hline $\mathrm{O}(16)-\mathrm{W}(1)-\mathrm{O}(21)$ & $102.7(4)$ & $\mathrm{O}(40)-\mathrm{W}(15)-\mathrm{O}(29)$ & $86.6(4)$ \\
\hline $\mathrm{O}(16)-\mathrm{W}(1)-\mathrm{O}(20)$ & $100.6(5)$ & $\mathrm{O}(39)-\mathrm{W}(15)-\mathrm{O}(29)$ & $87.3(4)$ \\
\hline $\mathrm{O}(21)-\mathrm{W}(1)-\mathrm{O}(20)$ & $87.3(4)$ & $\mathrm{O}(4)-\mathrm{W}(15)-\mathrm{O}(15)$ & $171.4(5)$ \\
\hline $\mathrm{O}(16)-\mathrm{W}(1)-\mathrm{O}(25)$ & $101.5(5)$ & $\mathrm{O}(28)-\mathrm{W}(15)-\mathrm{O}(15)$ & $82.8(3)$ \\
\hline $\mathrm{O}(21)-\mathrm{W}(1)-\mathrm{O}(25)$ & $155.8(4)$ & $\mathrm{O}(40)-\mathrm{W}(15)-\mathrm{O}(15)$ & $84.6(3)$ \\
\hline $\mathrm{O}(20)-\mathrm{W}(1)-\mathrm{O}(25)$ & $88.5(4)$ & $\mathrm{O}(39)-\mathrm{W}(15)-\mathrm{O}(15)$ & $72.7(3)$ \\
\hline $\mathrm{O}(16)-\mathrm{W}(1)-\mathrm{O}(24)$ & $101.7(5)$ & $\mathrm{O}(29)-\mathrm{W}(15)-\mathrm{O}(15)$ & $79.7(3)$ \\
\hline $\mathrm{O}(21)-\mathrm{W}(1)-\mathrm{O}(24)$ & $88.9(4)$ & $\mathrm{O}(5)-\mathrm{W}(16)-\mathrm{O}(19)$ & $99.5(5)$ \\
\hline $\mathrm{O}(20)-\mathrm{W}(1)-\mathrm{O}(24)$ & $157.6(4)$ & $\mathrm{O}(5)-\mathrm{W}(16)-\mathrm{O}(34)$ & $104.0(5)$ \\
\hline $\mathrm{O}(25)-\mathrm{W}(1)-\mathrm{O}(24)$ & $86.0(4)$ & $\mathrm{O}(19)-\mathrm{W}(16)-\mathrm{O}(34)$ & $86.4(4)$ \\
\hline $\mathrm{O}(16)-\mathrm{W}(1)-\mathrm{O}(46)$ & $171.9(4)$ & $\mathrm{O}(5)-\mathrm{W}(16)-\mathrm{O}(54)$ & $100.6(5)$ \\
\hline $\mathrm{O}(21)-\mathrm{W}(1)-\mathrm{O}(46)$ & $83.8(3)$ & $\mathrm{O}(19)-\mathrm{W}(16)-\mathrm{O}(54)$ & $89.8(4)$ \\
\hline $\mathrm{O}(20)-\mathrm{W}(1)-\mathrm{O}(46)$ & $84.4(4)$ & $\mathrm{O}(34)-\mathrm{W}(16)-\mathrm{O}(54)$ & $155.4(4)$ \\
\hline $\mathrm{O}(25)-\mathrm{W}(1)-\mathrm{O}(46)$ & $72.1(4)$ & $\mathrm{O}(5)-\mathrm{W}(16)-\mathrm{O}(33)$ & $97.9(5)$ \\
\hline $\mathrm{O}(24)-\mathrm{W}(1)-\mathrm{O}(46)$ & $73.3(4)$ & $\mathrm{O}(19)-\mathrm{W}(16)-\mathrm{O}(33)$ & $162.1(4)$ \\
\hline $\mathrm{O}(59)-\mathrm{W}(2)-\mathrm{O}(25)$ & $102.6(5)$ & $\mathrm{O}(34)-\mathrm{W}(16)-\mathrm{O}(33)$ & $85.7(4)$ \\
\hline $\mathrm{O}(59)-\mathrm{W}(2)-\mathrm{O}(48)$ & $102.0(4)$ & $\mathrm{O}(54)-\mathrm{W}(16)-\mathrm{O}(33)$ & $90.7(4)$ \\
\hline $\mathrm{O}(25)-\mathrm{W}(2)-\mathrm{O}(48)$ & $89.6(4)$ & $\mathrm{O}(5)-\mathrm{W}(16)-\mathrm{O}(38)$ & $173.6(5)$ \\
\hline $\mathrm{O}(59)-\mathrm{W}(2)-\mathrm{O}(22)$ & $101.8(4)$ & $\mathrm{O}(19)-\mathrm{W}(16)-\mathrm{O}(38)$ & $81.3(3)$ \\
\hline $\mathrm{O}(25)-\mathrm{W}(2)-\mathrm{O}(22)$ & $155.6(4)$ & $\mathrm{O}(34)-\mathrm{W}(16)-\mathrm{O}(38)$ & $82.4(4)$ \\
\hline $\mathrm{O}(48)-\mathrm{W}(2)-\mathrm{O}(22)$ & $85.5(4)$ & $\mathrm{O}(54)-\mathrm{W}(16)-\mathrm{O}(38)$ & $73.0(3)$ \\
\hline $\mathrm{O}(59)-\mathrm{W}(2)-\mathrm{O}(43)$ & $100.8(4)$ & $\mathrm{O}(33)-\mathrm{W}(16)-\mathrm{O}(38)$ & $81.8(3)$ \\
\hline $\mathrm{O}(25)-\mathrm{W}(2)-\mathrm{O}(43)$ & $87.8(4)$ & $\mathrm{O}(62)-\mathrm{W}(17)-\mathrm{O}(34)$ & $103.8(4)$ \\
\hline $\mathrm{O}(48)-\mathrm{W}(2)-\mathrm{O}(43)$ & 157.1(4) & $\mathrm{O}(62)-\mathrm{W}(17)-\mathrm{O}(44)$ & $97.4(5)$ \\
\hline $\mathrm{O}(22)-\mathrm{W}(2)-\mathrm{O}(43)$ & $87.5(4)$ & $\mathrm{O}(34)-\mathrm{W}(17)-\mathrm{O}(44)$ & $87.4(4)$ \\
\hline $\mathrm{O}(59)-\mathrm{W}(2)-\mathrm{O}(46)$ & $171.9(4)$ & $\mathrm{O}(62)-\mathrm{W}(17)-\mathrm{O}(18)$ & $97.5(5)$ \\
\hline $\mathrm{O}(25)-\mathrm{W}(2)-\mathrm{O}(46)$ & $72.2(4)$ & $\mathrm{O}(34)-\mathrm{W}(17)-\mathrm{O}(18)$ & $89.7(4)$ \\
\hline
\end{tabular}




\begin{tabular}{|c|c|c|c|}
\hline $\mathrm{O}(48)-\mathrm{W}(2)-\mathrm{O}(46)$ & $84.3(3)$ & $\mathrm{O}(44)-\mathrm{W}(17)-\mathrm{O}(18)$ & $165.0(4)$ \\
\hline $\mathrm{O}(22)-\mathrm{W}(2)-\mathrm{O}(46)$ & $83.5(3)$ & $\mathrm{O}(62)-\mathrm{W}(17)-\mathrm{O}(60)$ & $96.9(4)$ \\
\hline $\mathrm{O}(43)-\mathrm{W}(2)-\mathrm{O}(46)$ & $73.2(3)$ & $\mathrm{O}(34)-\mathrm{W}(17)-\mathrm{O}(60)$ & $159.2(4)$ \\
\hline $\mathrm{O}(17)-\mathrm{W}(3)-\mathrm{O}(24)$ & $101.3(5)$ & $\mathrm{O}(44)-\mathrm{W}(17)-\mathrm{O}(60)$ & $89.0(4)$ \\
\hline $\mathrm{O}(17)-\mathrm{W}(3)-\mathrm{O}(52)$ & $102.3(4)$ & $\mathrm{O}(18)-\mathrm{W}(17)-\mathrm{O}(60)$ & $88.6(4)$ \\
\hline $\mathrm{O}(24)-\mathrm{W}(3)-\mathrm{O}(52)$ & $90.0(4)$ & $\mathrm{O}(62)-\mathrm{W}(17)-\mathrm{O}(50)$ & $170.8(4)$ \\
\hline $\mathrm{O}(17)-\mathrm{W}(3)-\mathrm{O}(23)$ & $101.0(5)$ & $\mathrm{O}(34)-\mathrm{W}(17)-\mathrm{O}(50)$ & $85.3(4)$ \\
\hline $\mathrm{O}(24)-\mathrm{W}(3)-\mathrm{O}(23)$ & $157.7(4)$ & $\mathrm{O}(44)-\mathrm{W}(17)-\mathrm{O}(50)$ & $82.5(4)$ \\
\hline $\mathrm{O}(52)-\mathrm{W}(3)-\mathrm{O}(23)$ & $85.2(4)$ & $\mathrm{O}(18)-\mathrm{W}(17)-\mathrm{O}(50)$ & $82.7(4)$ \\
\hline $\mathrm{O}(17)-\mathrm{W}(3)-\mathrm{O}(43)$ & $100.5(4)$ & $\mathrm{O}(60)-\mathrm{W}(17)-\mathrm{O}(50)$ & $73.9(3)$ \\
\hline $\mathrm{O}(24)-\mathrm{W}(3)-\mathrm{O}(43)$ & $88.8(4)$ & $\mathrm{O}(6)-\mathrm{W}(18)-\mathrm{O}(53)$ & $99.3(5)$ \\
\hline $\mathrm{O}(52)-\mathrm{W}(3)-\mathrm{O}(43)$ & $156.9(4)$ & $\mathrm{O}(6)-\mathrm{W}(18)-\mathrm{O}(55)$ & $97.6(5)$ \\
\hline $\mathrm{O}(23)-\mathrm{W}(3)-\mathrm{O}(43)$ & $87.2(4)$ & $\mathrm{O}(53)-\mathrm{W}(18)-\mathrm{O}(55)$ & $162.4(4)$ \\
\hline $\mathrm{O}(17)-\mathrm{W}(3)-\mathrm{O}(46)$ & $172.0(5)$ & $\mathrm{O}(6)-\mathrm{W}(18)-\mathrm{O}(60)$ & $99.8(4)$ \\
\hline $\mathrm{O}(24)-\mathrm{W}(3)-\mathrm{O}(46)$ & $73.8(3)$ & $\mathrm{O}(53)-\mathrm{W}(18)-\mathrm{O}(60)$ & $91.6(4)$ \\
\hline $\mathrm{O}(52)-\mathrm{W}(3)-\mathrm{O}(46)$ & $84.2(3)$ & $\mathrm{O}(55)-\mathrm{W}(18)-\mathrm{O}(60)$ & $90.4(4)$ \\
\hline $\mathrm{O}(23)-\mathrm{W}(3)-\mathrm{O}(46)$ & $84.0(3)$ & $\mathrm{O}(6)-\mathrm{W}(18)-\mathrm{O}(40)$ & $102.6(4)$ \\
\hline $\mathrm{O}(43)-\mathrm{W}(3)-\mathrm{O}(46)$ & $73.3(3)$ & $\mathrm{O}(53)-\mathrm{W}(18)-\mathrm{O}(40)$ & $85.4(4)$ \\
\hline $\mathrm{O}(12)-\mathrm{W}(4)-\mathrm{O}(58)$ & $102.5(5)$ & $\mathrm{O}(55)-\mathrm{W}(18)-\mathrm{O}(40)$ & $86.2(4)$ \\
\hline $\mathrm{O}(12)-\mathrm{W}(4)-\mathrm{O}(49)$ & $99.7(5)$ & $\mathrm{O}(60)-\mathrm{W}(18)-\mathrm{O}(40)$ & $157.7(4)$ \\
\hline $\mathrm{O}(58)-\mathrm{W}(4)-\mathrm{O}(49)$ & $157.8(4)$ & $\mathrm{O}(6)-\mathrm{W}(18)-\mathrm{O}(50)$ & $173.1(4)$ \\
\hline $\mathrm{O}(12)-\mathrm{W}(4)-\mathrm{O}(52)$ & $98.6(4)$ & $\mathrm{O}(53)-\mathrm{W}(18)-\mathrm{O}(50)$ & $82.5(3)$ \\
\hline $\mathrm{O}(58)-\mathrm{W}(4)-\mathrm{O}(52)$ & $86.1(4)$ & $\mathrm{O}(55)-\mathrm{W}(18)-\mathrm{O}(50)$ & $81.3(4)$ \\
\hline $\mathrm{O}(49)-\mathrm{W}(4)-\mathrm{O}(52)$ & $89.7(4)$ & $\mathrm{O}(60)-\mathrm{W}(18)-\mathrm{O}(50)$ & $73.4(3)$ \\
\hline $\mathrm{O}(12)-\mathrm{W}(4)-\mathrm{O}(56)$ & $97.6(5)$ & $\mathrm{O}(40)-\mathrm{W}(18)-\mathrm{O}(50)$ & $84.2(3)$ \\
\hline $\mathrm{O}(58)-\mathrm{W}(4)-\mathrm{O}(56)$ & $87.9(4)$ & $\mathrm{O}(3)-\mathrm{W}(11)-\mathrm{O}(29)$ & $102.3(4)$ \\
\hline $\mathrm{O}(49)-\mathrm{W}(4)-\mathrm{O}(56)$ & $90.1(4)$ & $\mathrm{O}(3)-\mathrm{W}(11)-\mathrm{O}(37)$ & $101.7(5)$ \\
\hline $\mathrm{O}(52)-\mathrm{W}(4)-\mathrm{O}(56)$ & $163.6(4)$ & $\mathrm{O}(29)-\mathrm{W}(11)-\mathrm{O}(37)$ & $88.4(4)$ \\
\hline $\mathrm{O}(12)-\mathrm{W}(4)-\mathrm{O}(51)$ & $172.7(4)$ & $\mathrm{O}(3)-\mathrm{W}(11)-\mathrm{O}(31)$ & $101.9(4)$ \\
\hline $\mathrm{O}(58)-\mathrm{W}(4)-\mathrm{O}(51)$ & $84.8(3)$ & $\mathrm{O}(29)-\mathrm{W}(11)-\mathrm{O}(31)$ & $155.4(4)$ \\
\hline $\mathrm{O}(49)-\mathrm{W}(4)-\mathrm{O}(51)$ & $73.1(3)$ & $\mathrm{O}(37)-\mathrm{W}(11)-\mathrm{O}(31)$ & $90.6(5)$ \\
\hline $\mathrm{O}(52)-\mathrm{W}(4)-\mathrm{O}(51)$ & $81.7(3)$ & $\mathrm{O}(3)-\mathrm{W}(11)-\mathrm{O}(55)$ & $101.0(5)$ \\
\hline $\mathrm{O}(56)-\mathrm{W}(4)-\mathrm{O}(51)$ & $82.6(4)$ & $\mathrm{O}(29)-\mathrm{W}(11)-\mathrm{O}(55)$ & $86.0(4)$ \\
\hline $\mathrm{O}(13)-\mathrm{W}(5)-\mathrm{O}(47)$ & $100.1(4)$ & $\mathrm{O}(37)-\mathrm{W}(11)-\mathrm{O}(55)$ & $157.3(4)$ \\
\hline $\mathrm{O}(13)-\mathrm{W}(5)-\mathrm{O}(23)$ & $97.7(5)$ & $\mathrm{O}(31)-\mathrm{W}(11)-\mathrm{O}(55)$ & $85.5(5)$ \\
\hline $\mathrm{O}(47)-\mathrm{W}(5)-\mathrm{O}(23)$ & $91.4(4)$ & $\mathrm{O}(3)-\mathrm{W}(11)-\mathrm{O}(35)$ & $173.2(4)$ \\
\hline $\mathrm{O}(13)-\mathrm{W}(5)-\mathrm{O}(58)$ & $101.3(4)$ & $\mathrm{O}(29)-\mathrm{W}(11)-\mathrm{O}(35)$ & $83.1(3)$ \\
\hline $\mathrm{O}(47)-\mathrm{W}(5)-\mathrm{O}(58)$ & $158.6(4)$ & $\mathrm{O}(37)-\mathrm{W}(11)-\mathrm{O}(35)$ & $74.1(4)$ \\
\hline $\mathrm{O}(23)-\mathrm{W}(5)-\mathrm{O}(58)$ & $87.1(4)$ & $\mathrm{O}(31)-\mathrm{W}(11)-\mathrm{O}(35)$ & $73.0(3)$ \\
\hline $\mathrm{O}(13)-\mathrm{W}(5)-\mathrm{O}(27)$ & $97.3(5)$ & $\mathrm{O}(55)-\mathrm{W}(11)-\mathrm{O}(35)$ & $83.4(4)$ \\
\hline $\mathrm{O}(47)-\mathrm{W}(5)-\mathrm{O}(27)$ & $88.1(4)$ & $\mathrm{O}(1)-\mathrm{W}(12)-\mathrm{O}(44)$ & $102.1(5)$ \\
\hline $\mathrm{O}(23)-\mathrm{W}(5)-\mathrm{O}(27)$ & $164.9(4)$ & $\mathrm{O}(1)-\mathrm{W}(12)-\mathrm{O}(33)$ & $101.9(4)$ \\
\hline $\mathrm{O}(58)-\mathrm{W}(5)-\mathrm{O}(27)$ & $87.8(4)$ & $\mathrm{O}(44)-\mathrm{W}(12)-\mathrm{O}(33)$ & $85.0(4)$ \\
\hline
\end{tabular}




\begin{tabular}{|c|c|c|c|}
\hline $\mathrm{O}(13)-\mathrm{W}(5)-\mathrm{O}(42)$ & $173.1(4)$ & $\mathrm{O}(1)-\mathrm{W}(12)-\mathrm{O}(31)$ & $102.3(5)$ \\
\hline $\mathrm{O}(47)-\mathrm{W}(5)-\mathrm{O}(42)$ & $73.0(3)$ & $\mathrm{O}(44)-\mathrm{W}(12)-\mathrm{O}(31)$ & $86.6(5)$ \\
\hline $\mathrm{O}(23)-\mathrm{W}(5)-\mathrm{O}(42)$ & $82.5(4)$ & $\mathrm{O}(33)-\mathrm{W}(12)-\mathrm{O}(31)$ & $155.5(4)$ \\
\hline $\mathrm{O}(58)-\mathrm{W}(5)-\mathrm{O}(42)$ & $85.6(3)$ & $\mathrm{O}(1)-\mathrm{W}(12)-\mathrm{O}(32)$ & $102.2(5)$ \\
\hline $\mathrm{O}(27)-\mathrm{W}(5)-\mathrm{O}(42)$ & $82.9(4)$ & $\mathrm{O}(44)-\mathrm{W}(12)-\mathrm{O}(32)$ & $155.6(4)$ \\
\hline $\mathrm{O}(10)-\mathrm{W}(6)-\mathrm{O}(26)$ & $103.0(4)$ & $\mathrm{O}(33)-\mathrm{W}(12)-\mathrm{O}(32)$ & $87.7(4)$ \\
\hline $\mathrm{O}(10)-\mathrm{W}(6)-\mathrm{O}(47)$ & $100.9(4)$ & $\mathrm{O}(31)-\mathrm{W}(12)-\mathrm{O}(32)$ & $90.6(4)$ \\
\hline $\mathrm{O}(26)-\mathrm{W}(6)-\mathrm{O}(47)$ & $156.2(4)$ & $\mathrm{O}(1)-\mathrm{W}(12)-\mathrm{O}(35)$ & $172.5(5)$ \\
\hline $\mathrm{O}(10)-\mathrm{W}(6)-\mathrm{O}(28)$ & $98.3(4)$ & $\mathrm{O}(44)-\mathrm{W}(12)-\mathrm{O}(35)$ & $83.2(4)$ \\
\hline $\mathrm{O}(26)-\mathrm{W}(6)-\mathrm{O}(28)$ & $87.2(4)$ & $\mathrm{O}(33)-\mathrm{W}(12)-\mathrm{O}(35)$ & $83.8(3)$ \\
\hline $\mathrm{O}(47)-\mathrm{W}(6)-\mathrm{O}(28)$ & $89.9(4)$ & $\mathrm{O}(31)-\mathrm{W}(12)-\mathrm{O}(35)$ & $72.4(3)$ \\
\hline $\mathrm{O}(10)-\mathrm{W}(6)-\mathrm{O}(22)$ & $98.3(4)$ & $\mathrm{O}(32)-\mathrm{W}(12)-\mathrm{O}(35)$ & $72.9(4)$ \\
\hline $\mathrm{O}(26)-\mathrm{W}(6)-\mathrm{O}(22)$ & $85.6(4)$ & $\mathrm{O}(8)-\mathrm{W}(13)-\mathrm{O}(27)$ & $98.3(5)$ \\
\hline $\mathrm{O}(47)-\mathrm{W}(6)-\mathrm{O}(22)$ & $90.5(4)$ & $\mathrm{O}(8)-\mathrm{W}(13)-\mathrm{O}(39)$ & $99.9(4)$ \\
\hline $\mathrm{O}(28)-\mathrm{W}(6)-\mathrm{O}(22)$ & $163.0(3)$ & $\mathrm{O}(27)-\mathrm{W}(13)-\mathrm{O}(39)$ & $92.5(4)$ \\
\hline $\mathrm{O}(10)-\mathrm{W}(6)-\mathrm{O}(42)$ & $172.8(4)$ & $\mathrm{O}(8)-\mathrm{W}(13)-\mathrm{O}(45)$ & $101.1(4)$ \\
\hline $\mathrm{O}(26)-\mathrm{W}(6)-\mathrm{O}(42)$ & $84.0(3)$ & $\mathrm{O}(27)-\mathrm{W}(13)-\mathrm{O}(45)$ & $87.8(4)$ \\
\hline $\mathrm{O}(47)-\mathrm{W}(6)-\mathrm{O}(42)$ & $72.2(3)$ & $\mathrm{O}(39)-\mathrm{W}(13)-\mathrm{O}(45)$ & $158.7(4)$ \\
\hline $\mathrm{O}(28)-\mathrm{W}(6)-\mathrm{O}(42)$ & $83.6(3)$ & $\mathrm{O}(8)-\mathrm{W}(13)-\mathrm{O}(30)$ & $96.0(5)$ \\
\hline $\mathrm{O}(22)-\mathrm{W}(6)-\mathrm{O}(42)$ & $80.4(3)$ & $\mathrm{O}(27)-\mathrm{W}(13)-\mathrm{O}(30)$ & $164.7(4)$ \\
\hline $\mathrm{O}(9)-\mathrm{W}(7)-\mathrm{O}(26)$ & $102.4(4)$ & $\mathrm{O}(39)-\mathrm{W}(13)-\mathrm{O}(30)$ & $90.4(4)$ \\
\hline $\mathrm{O}(9)-\mathrm{W}(7)-\mathrm{O}(57)$ & $100.5(4)$ & $\mathrm{O}(45)-\mathrm{W}(13)-\mathrm{O}(30)$ & $84.1(4)$ \\
\hline $\mathrm{O}(26)-\mathrm{W}(7)-\mathrm{O}(57)$ & $157.0(4)$ & $\mathrm{O}(8)-\mathrm{W}(13)-\mathrm{O}(15)$ & $174.0(4)$ \\
\hline $\mathrm{O}(9)-\mathrm{W}(7)-\mathrm{O}(53)$ & 98.3(4) & $\mathrm{O}(27)-\mathrm{W}(13)-\mathrm{O}(15)$ & $84.3(4)$ \\
\hline $\mathrm{O}(26)-\mathrm{W}(7)-\mathrm{O}(53)$ & $88.7(4)$ & $\mathrm{O}(39)-\mathrm{W}(13)-\mathrm{O}(15)$ & $74.6(4)$ \\
\hline $\mathrm{O}(57)-\mathrm{W}(7)-\mathrm{O}(53)$ & $90.1(4)$ & $\mathrm{O}(14)-\mathrm{W}(9)-\mathrm{O}(19)$ & $99.4(4)$ \\
\hline $\mathrm{O}(9)-\mathrm{W}(7)-\mathrm{O}(48)$ & $97.7(4)$ & $\mathrm{O}(14)-\mathrm{W}(9)-\mathrm{O}(49)$ & $98.6(5)$ \\
\hline $\mathrm{O}(26)-\mathrm{W}(7)-\mathrm{O}(48)$ & $85.7(4)$ & $\mathrm{O}(19)-\mathrm{W}(9)-\mathrm{O}(49)$ & $91.0(4)$ \\
\hline $\mathrm{O}(57)-\mathrm{W}(7)-\mathrm{O}(48)$ & $89.3(4)$ & $\mathrm{O}(14)-\mathrm{W}(9)-\mathrm{O}(61)$ & $103.4(5)$ \\
\hline $\mathrm{O}(53)-\mathrm{W}(7)-\mathrm{O}(48)$ & $163.9(4)$ & $\mathrm{O}(19)-\mathrm{W}(9)-\mathrm{O}(61)$ & $90.7(4)$ \\
\hline $\mathrm{O}(9)-\mathrm{W}(7)-\mathrm{O}(41)$ & $172.8(4)$ & $\mathrm{O}(49)-\mathrm{W}(9)-\mathrm{O}(61)$ & $157.3(4)$ \\
\hline $\mathrm{O}(26)-\mathrm{W}(7)-\mathrm{O}(41)$ & $84.6(3)$ & $\mathrm{O}(14)-\mathrm{W}(9)-\mathrm{O}(21)$ & $97.3(4)$ \\
\hline $\mathrm{O}(57)-\mathrm{W}(7)-\mathrm{O}(41)$ & $72.4(4)$ & $\mathrm{O}(19)-\mathrm{W}(9)-\mathrm{O}(21)$ & $163.2(4)$ \\
\hline $\mathrm{O}(53)-\mathrm{W}(7)-\mathrm{O}(41)$ & $83.3(3)$ & $\mathrm{O}(49)-\mathrm{W}(9)-\mathrm{O}(21)$ & $87.8(4)$ \\
\hline $\mathrm{O}(48)-\mathrm{W}(7)-\mathrm{O}(41)$ & $81.1(3)$ & $\mathrm{O}(61)-\mathrm{W}(9)-\mathrm{O}(21)$ & $84.1(4)$ \\
\hline $\mathrm{O}(11)-\mathrm{W}(8)-\mathrm{O}(61)$ & $101.6(5)$ & $\mathrm{O}(14)-\mathrm{W}(9)-\mathrm{O}(51)$ & $170.8(4)$ \\
\hline $\mathrm{O}(11)-\mathrm{W}(8)-\mathrm{O}(18)$ & $98.6(4)$ & $\mathrm{O}(19)-\mathrm{W}(9)-\mathrm{O}(51)$ & $83.7(4)$ \\
\hline $\mathrm{O}(61)-\mathrm{W}(8)-\mathrm{O}(18)$ & $89.2(4)$ & $\mathrm{O}(49)-\mathrm{W}(9)-\mathrm{O}(51)$ & $72.5(3)$ \\
\hline $\mathrm{O}(11)-\mathrm{W}(8)-\mathrm{O}(57)$ & $100.4(5)$ & $\mathrm{O}(61)-\mathrm{W}(9)-\mathrm{O}(51)$ & $85.2(4)$ \\
\hline $\mathrm{O}(61)-\mathrm{W}(8)-\mathrm{O}(57)$ & $157.9(4)$ & $\mathrm{O}(21)-\mathrm{W}(9)-\mathrm{O}(51)$ & $80.0(3)$ \\
\hline $\mathrm{O}(18)-\mathrm{W}(8)-\mathrm{O}(57)$ & $89.7(4)$ & $\mathrm{O}(2)-\mathrm{W}(10)-\mathrm{O}(36)$ & $101.7(5)$ \\
\hline $\mathrm{O}(11)-\mathrm{W}(8)-\mathrm{O}(20)$ & $97.8(4)$ & $\mathrm{O}(2)-\mathrm{W}(10)-\mathrm{O}(30)$ & $101.7(5)$ \\
\hline $\mathrm{O}(61)-\mathrm{W}(8)-\mathrm{O}(20)$ & $84.9(4)$ & $\mathrm{O}(36)-\mathrm{W}(10)-\mathrm{O}(30)$ & $86.1(4)$ \\
\hline
\end{tabular}




\begin{tabular}{|l|l|l|l|}
\hline $\mathrm{O}(18)-\mathrm{W}(8)-\mathrm{O}(20)$ & $163.4(4)$ & $\mathrm{O}(2)-\mathrm{W}(10)-\mathrm{O}(32)$ & $101.7(5)$ \\
\hline $\mathrm{O}(57)-\mathrm{W}(8)-\mathrm{O}(20)$ & $90.0(4)$ & $\mathrm{O}(36)-\mathrm{W}(10)-\mathrm{O}(32)$ & $88.8(4)$ \\
\hline $\mathrm{O}(11)-\mathrm{W}(8)-\mathrm{O}(41)$ & $173.4(4)$ & $\mathrm{O}(30)-\mathrm{W}(10)-\mathrm{O}(32)$ & $156.6(5)$ \\
\hline $\mathrm{O}(61)-\mathrm{W}(8)-\mathrm{O}(41)$ & $85.0(4)$ & $\mathrm{O}(2)-\mathrm{W}(10)-\mathrm{O}(37)$ & $100.8(5)$ \\
\hline $\mathrm{O}(18)-\mathrm{W}(8)-\mathrm{O}(41)$ & $82.6(4)$ & $\mathrm{O}(36)-\mathrm{W}(10)-\mathrm{O}(37)$ & $157.5(5)$ \\
\hline $\mathrm{O}(57)-\mathrm{W}(8)-\mathrm{O}(41)$ & $73.0(4)$ & $\mathrm{O}(30)-\mathrm{W}(10)-\mathrm{O}(37)$ & $87.2(4)$ \\
\hline $\mathrm{O}(20)-\mathrm{W}(8)-\mathrm{O}(41)$ & $81.5(3)$ & $\mathrm{O}(32)-\mathrm{W}(10)-\mathrm{O}(37)$ & $88.8(4)$ \\
\hline $\mathrm{O}(32)-\mathrm{W}(10)-\mathrm{O}(35)$ & $73.7(4)$ & $\mathrm{O}(2)-\mathrm{W}(10)-\mathrm{O}(35)$ & $172.1(4)$ \\
\hline
\end{tabular}

